\title{
Characteristic space scales and timescales in hydrology
}

\author{
J. O. Skøien and G. Blöschl \\ Institut für Hydraulik, Gewässerkunde und Wasserwirtschaft, Technische Universität Wien, Vienna, Austria
}

\begin{abstract}
A. W. Western
Centre for Environmental Applied Hydrology and Cooperative Research Centre for Catchment Hydrology, Department of Civil and Environmental Engineering, University of Melbourne, Parkville, Victoria, Australia
\end{abstract}

Received 23 September 2002; revised 1 July 2003; accepted 7 August 2003; published 30 October 2003.

[1] We analyzed spatial and temporal variograms of precipitation, runoff, and groundwater levels in Austria to examine whether characteristic scales exist and, if so, how big they are. In time, precipitation and runoff are stationary with characteristic scales on the order of a day and a month, respectively, while groundwater levels are nonstationary. In space, precipitation is almost fractal, so no characteristic scales exist. Runoff is nonstationary but not a fractal as it exhibits a break in the variograms. An analysis of the variograms of catchment precipitation indicates that this break is due to aggregation effects imposed by the catchment area. A spatial variogram of hypothetical point runoff back calculated from runoff variograms of three catchment size classes using aggregation statistics (regularization) is almost stationary and exhibits shorter characteristic space scales than catchment runoff. Groundwater levels are nonstationary in space, exhibiting shorter-scale variability than precipitation and runoff, but are also not fractal as there is a break in the variogram. We suggest that the decrease of spatial characteristic scales from catchment precipitation to runoff and to groundwater is the result of a superposition of small-scale variability of catchment and aquifer properties on the rainfall forcing. For comparison, TDR soil moisture data from a comprehensive Australian data set were examined. These data suggest that in time, soil moisture is close to stationary with characteristic scales of the order of 2 weeks while in space soil moisture is nonstationary and close to fractal over the extent sampled. Space-time traces of characteristic scales fit well into a conceptual diagram of Blöschl and Sivapalan [1995]. The scaling exponents $z$ in $T \sim L^{\mathrm{Z}}$ (where $T$ is time and $L$ is space) are of the order of 0.5 for precipitation, 0.8 for runoff from small catchments, 1.2 for runoff from large catchments, 0.8 for groundwater levels, and 0.5 for soil moisture. INDEX TERMS: 1829 Hydrology: Groundwater hydrology; 1854 Hydrology: Precipitation (3354); 1860 Hydrology: Runoff and streamflow; 1866 Hydrology: Soil moisture; 1869 Hydrology: Stochastic processes; KEYWORDS: variogram, fractal, stationarity, aggregation

Citation: Skøien, J. O., G. Blöschl, and A. W. Western, Characteristic space scales and timescales in hydrology, Water Resour. Res., 39(10), 1304, doi:10.1029/2002WR001736, 2003.

\section{Introduction}

[2] Variability of hydrologic processes occurs over many orders of magnitude, from pore-scale flow processes up to the global cycling of water and energy, from rain splash effects during less than a second up to changes in the hydrologic balance over centuries and more as a result of climate fluctuations and geomorphic processes. The wide range of variability complicates the scientific analysis of hydrologic processes, be it through theoretical analyses, measurements, or model studies. One concept of dealing with variability over many orders of magnitude is the notion of characteristic scales. The idea of a characteristic scale is that instead of dealing with a spectrum of lengths and times one adopts one typical length and time that is representative of a particular process. Often a characteristic scale is an order of magnitude figure, given as an integer power of ten, rather than a precise number.

Copyright 2003 by the American Geophysical Union. 0043-1397/03/2002WR001736
[3] While sister disciplines have an excellent track record of capitalizing on the potential of characteristic scales, their use in hydrology has been rare, even though their merits are undisputed [Dooge, 1986; Seyfried and Wilcox, 1995; Blöschl, 1999]. In fluid dynamics, atmospheric sciences and ecology, characteristic scales are widely used to tag processes for a number of purposes. Some of the most obvious merits are summarized below.

[4] First, characteristic scales can be used for performing order of magnitude or "back of the envelope" analyses. Excellent examples in the context of mixing processes in open waters are given by Fischer et al. [1979] and a range of illustrative examples in the environmental sciences in general are given by Harte [1988]. Second, characteristic scales may facilitate the formulation of simple relationships. For example, Prandtl's [1925] suggestion of a "mixing length" allowed him to determine the mean velocity distribution of a fluid near the wall with the aid of only one empirical constant. Third, characteristic scales are very powerful when used in connection with dimensional anal- 
yses and dimensionless numbers. The classical example is the Froude number, which is composed of two characteristic velocities. A good overview for boundary layer meteorology is given by Stull [1988] and a number of suggestions for how to apply dimensional analyses to hydrology are given by Dooge [1986]. Fourth, in a similar fashion, characteristic scales play a central role in similarity theories which are well developed in the atmospheric sciences [e.g., Haltiner and Williams, 1980], based on the early work of Charney [1948], and other disciplines such as soil physics [Miller and Miller, 1956]. In hydrology, similarity theories are at a very early stage of development [Blöschl and Sivapalan, 1995]. Fifth, characteristic scales are among the main input variables into behavioral or structure-imitating space-time models [e.g., Rodríguez-Iturbe, 1986; Koltermann and Gorelick, 1996]. Sixth, characteristic scales provide a powerful tool for planning and interpreting measurements. In order to capture a particular process the space-time scales of the measurement set up must be compatible with the characteristic scales of the processes one is attempting to sample. The case is excellently made by Smagorinsky [1974] for the example of ocean dynamics and an example in the context of the spatial distribution of soil moisture is given by Western and Blöschl [1999]. Lastly, and maybe most importantly, the identification of characteristic temporal and spatial scales relevant to a prescribed problem can provide a common framework between disciplines [Blöschl, 2001], as excellently illustrated by Seyfried and Wilcox [1995] for mountain hydrology and Hatcher et al. [1987] for the case of coral reef systems.

[5] In hydrology the various applications of characteristic scales are still in their infancy but it is hoped that deriving characteristic scales will foster the development of methods similar to those in the sister disciplines. Characteristic scales can be defined in a number of ways, either as deterministic scales or as stochastic scales [Blöschl, 1996]. Deterministic characteristic scales of hydrologic processes include storm size and storm duration [e.g., Austin and Houze, 1972]; size of saturation areas; residence times and response times from catchments and hillslopes [Uchida et al., 2001]; hillslope length and drainage density; and soil depth and aquifer depth. An alternative is to derive stochastic characteristic scales from a correlation analysis. Stochastic characteristic scales were first proposed by Taylor [1921] as a measure of the average correlation distance in turbulence and have since then been widely used for characterizing space-time variability in a number of disciplines [e.g., Vanmarcke, 1983; Christakos, 2000].

[6] In this paper we examine stochastic characteristic scales based on correlation analyses in both space and time. The correlation can be represented by the variogram (see, e.g., Webster and Oliver [2002] for a practical assessment). If the variable under study is stationary in the mean the variogram will flatten out at large distances while for nonstationary variables the variogram will increase continually. Strictly speaking, a characteristic scale only exists for a stationary variable and can be defined as the distance (or time) where the variogram flattens out. When deriving quantitative estimates of the characteristic scale from the variogram one can use one of various definitions of scale such as the integral scale (average distance of correlation), the range (the maximum distance of correlation), and the $e$-folding distance (the distance where the variogram value is $1-1 / e$ of its maximum value) [Blöschl, 1996] which all give similar orders of magnitude of the characteristic scale. For nonstationary variables, approximate characteristic scales can be derived if the variogram becomes flatter with increasing distance. If the variogram increases as a power of distance a characteristic scale does not exist, but this is another important case, termed a fractal variogram. It is of the form:

$$
\gamma(h)=a h^{Z}
$$

where $h$ is the lag (i.e., either distance or time lag), $a$ is a parameter controlling the variance, and $Z$ is the fractal power of the variogram. $Z$ is a measure of the ratio of large-scale variability and small-scale variability. Fractal behavior is an indication of long term persistence (or large spatial correlations) also referred to as the Hurst phenomenon [Klemeš, 1974; Kirchner et al., 2000]. A variogram of the form of equation (1) can be considered self similar as it satisfies

$$
\gamma(h)=\lambda^{-Z} \gamma(\lambda h)
$$

where $\lambda$ is a scale factor, i.e., lag dependent variance at one scale is a constant multiple of that at another scale, and this constant depends on the scale factor. Because of the self similarity property, fractal variability lends itself to similarity analyses (alternatively to the use of characteristic scales) and many of the other analyses that can be done with characteristic scales. It is therefore useful to ascertain whether characteristic scales exists, and if not, if the variogram is fractal and what the fractal power is. It is difficult to accurately determine the fractal power $Z$ [Gallant et al., $1994]$ and to ascertain with certainty whether the variogram is fractal or not [Kirchner et al., 2000] so a reliable analysis must be based on a sufficiently large data set.

[7] Characteristic timescales can be derived from a variogram analysis of time series. Characteristic space scales can be derived from a variogram analysis of spatial patterns. It is also of interest to examine the space-time behavior of characteristic scales. Stommel [1963] was probably the first to introduce diagrams showing a schematic relationship between spatial and temporal process scales. He used the diagram for characterizing ocean dynamics. Blöschl and Sivapalan [1995] adapted the idea to hydrology and based their Figure 2 on data from Orlanski [1975], Dunne [1978], Fortak [1982], and Anderson and Burt [1990] as well as heuristic reasoning. Their diagram has been widely used in the literature to illustrate the existence of characteristic space-time scales in hydrology in a qualitative way [e.g., Wilby, 1997; Quattrochi, 1997; Ambroise, 1999; Buchholz, 2000; Niehoff, 2002; Schulze, 2000]. In this type of diagram two quantities are of main interest. The first is the characteristic velocity of a process defined as the ratio of characteristic lengths and times. The characteristic velocity is a measure of the scales over which measurements become decorrelated from one another and may be related to a number of physical causes. It may be related to the celerity with which a perturbation (such as a weather front) moves through a system. It may correspond to particle velocities (such as typical vertical velocities in convective cells), and it may be related to the internal dispersion and disruption by smaller-scale perturbations. Blöschl and Sivapalan [1995] and Blöschl et al. [1995] suggested that typically, charac- 
teristic velocities are on the order of $10 \mathrm{~m} / \mathrm{s}$ for rain processes, $1 \mathrm{~m} / \mathrm{s}$ for channel flow, $0.1 \mathrm{~m} / \mathrm{s}$ for saturation excess overland flow and less than $10^{-4} \mathrm{~m} / \mathrm{s}$ for groundwater flow. The second quantity is the slope of the spacetime traces on a double logarithmic plot, i.e., the scaling exponent $z$ in

$$
T=a L^{\mathrm{z}}
$$

where $T$ is timescale, $L$ is space scale, and $a$ is a parameter. In Blöschl and Sivapalan's [1995] diagram, $z$ is about 0.9 for most processes which implies slightly increasing velocities with scale. They argued that these may result from a slight tendency of channel flow velocities to increase with catchments scale [Leopold and Maddock, 1953] and the behavior of response times for catchments of different sizes [Anderson and Burt, 1990]. It is interesting to compare the diagram of Blöschl and Sivapalan [1995] to a quantitative analysis of space-time rainfall variability of Foufoula-Georgiou and Vuruputur [2000] based on radar images of individual rainstorms. They examined fluctuations of precipitation in time and calculated standard deviations in space for different aggregation levels in space, and derived space-time pairs of constant standard deviations. To these pairs they fitted a relationship of the form of equation (3) and obtained values of $z=0.5-0.6$, i.e., significantly smaller values than those of Blöschl and Sivapalan [1995].

[8] While characteristic scales are usually defined for point processes in the literature it is also possible to examine characteristic scales of aggregated processes by treating runoff from catchments of a given size as a process associated with a particular characteristic scale. Provided runoff can be assumed to be a spatially homogeneous process the characteristic scale will be intrinsic to one particular catchment size but may be different for different catchment sizes. It is interesting to examine the effect catchment size has on characteristic scales, i.e., the effect of upscaling from small to large catchments. As one moves up in catchment scale, more and more spatial rainfall variability tends to get averaged out due to aggregation effects which will be reflected in a reduction of the variance of runoff and a tendency for increased characteristic scales [Western and Blöschl, 1999]. As one moves up in catchment scale, it is also likely that temporal variability decreases and characteristic temporal scales increase because of longer flow paths. However, as with other hydrologic characteristics such as extreme precipitation and floods, it is not quite clear what controls this variance reduction and the changes in characteristic scales as a function of catchment size [Blöschl and Sivapalan, 1997; Sivapalan and Blöschl, 1998]. It is important to unravel these controls in order to shed light on the space-time scaling behavior of catchments and to better understand the role of characteristic scales for catchments of different size.

[9] In the light of the above discussion the objectives of this paper are twofold. The first is to estimate characteristic space scales and timescales for the main variables of the hydrological cycle (precipitation, runoff, groundwater level, soil moisture) or, alternatively, identify their fractal characteristics if no characteristic scales exist. The second is to examine and understand the effect catchment size has on characteristic scales for the case of precipitation and runoff.

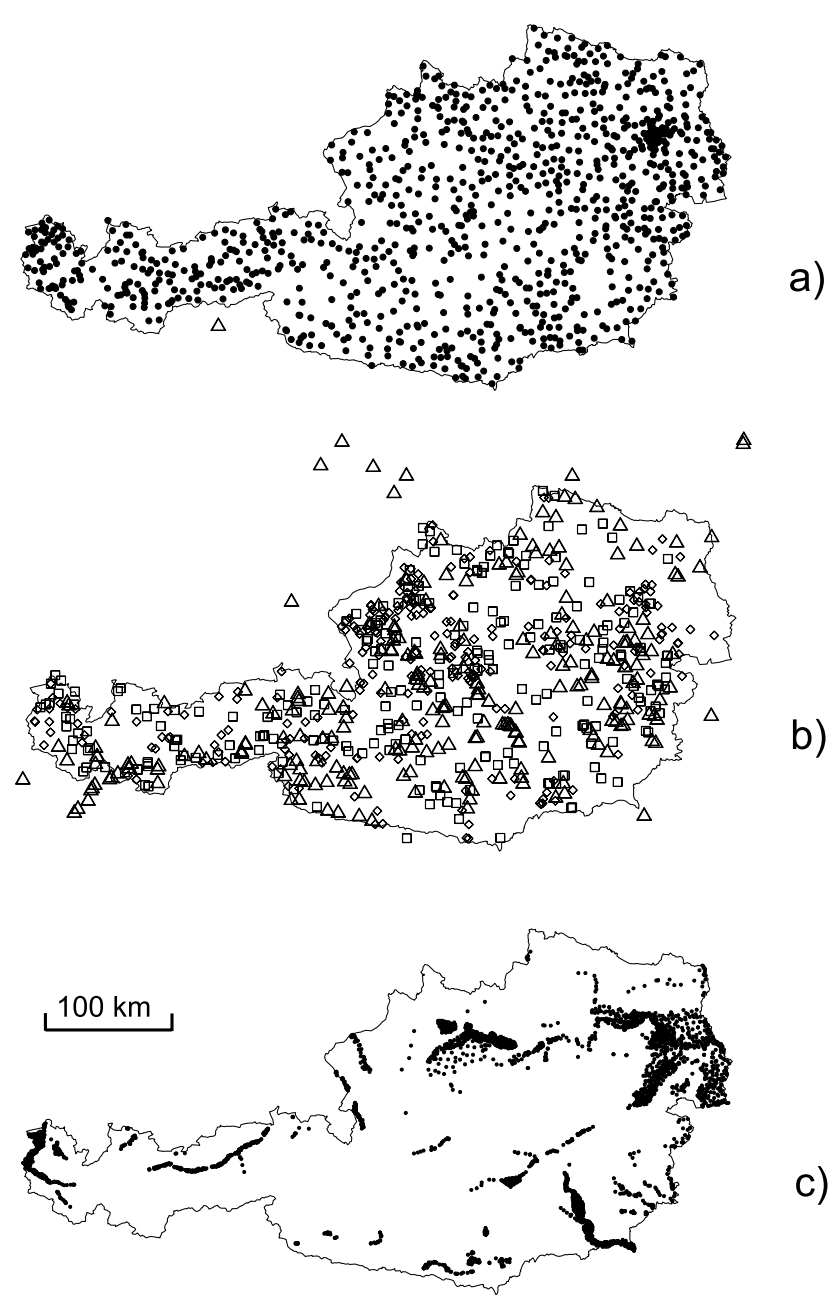

Figure 1. Network of measurement stations in Austria used in this paper. (a) Precipitation gauges. (b) Centroids of gauged catchments (small catchments shown as diamonds, medium sized catchments shown as squares, and large catchments shown as triangles). (c) Boreholes for groundwater levels.

Both objectives are addressed by extensive variogram analyses of hydrologic data.

\section{Data}

[10] Most of the data used in this paper stem from a comprehensive hydrographic data set of Austria. Austria has a varied climate with mean annual precipitation ranging from $500 \mathrm{~mm}$ in the eastern lowland regions up to about $3000 \mathrm{~mm}$ in the western alpine regions. Runoff depths range from less than $50 \mathrm{~mm}$ per year in the eastern part of the country to about $2000 \mathrm{~mm}$ per year in the Alps. Potential evapotranspiration is on the order of $600-900 \mathrm{~mm}$ per year. Mean daily runoff data for the period 1971-1997 were used. Stream gauges with short records and catchments with significant anthropogenic effects and lake effects were excluded from the data set which gave a total of 654 stream gauges available for the analysis. The runoff series were divided into three classes according to catchment size: small $\left(3-70 \mathrm{~km}^{2}\right)$, medium $\left(70-250 \mathrm{~km}^{2}\right)$, and large $(250-$ $130000 \mathrm{~km}^{2}$ ) (Figure 1b). The analyses were carried out for each class separately. Some of the catchments are nested. 
Table 1. Data Series Used in This Paper

\begin{tabular}{|c|c|c|c|c|c|c|c|}
\hline Data Type & Size Class & $\begin{array}{l}\text { Size Range, } \\
\mathrm{km}^{2}\end{array}$ & $\begin{array}{c}\text { Median Size, } \\
\mathrm{km}^{2}\end{array}$ & $\begin{array}{l}\text { Number of } \\
\text { Stations }\end{array}$ & $\begin{array}{c}\text { Extent of } \\
\text { Domain, } \mathrm{km}\end{array}$ & Time Resolution & Period \\
\hline Point precipitation & & point & point & 991 & 700 & daily sum & $1971-1997$ \\
\hline Catchment precipitation & small & $0.5-80$ & 36 & 252 & 700 & daily sum & $1971-1997$ \\
\hline Catchment precipitation & medium & $80-273$ & 135 & 252 & 700 & daily sum & $1971-1997$ \\
\hline Catchment precipitation & large & $277-131000$ & 818 & 252 & 700 & daily sum & $1971-1997$ \\
\hline Point precipitation & & point & point & 1 & - & hourly sum & $1988-1994$ \\
\hline Runoff & small & $2.8-74$ & 36 & 218 & 700 & daily average & $1971-1997$ \\
\hline Runoff & medium & $74-252$ & 128 & 218 & 700 & daily average & $1971-1997$ \\
\hline Runoff & large & $253-131000$ & 701 & 218 & 700 & daily average & $1971-1997$ \\
\hline Groundwater level & & point & point & 3539 & 700 & weekly instantaneous & $1966-1998$ \\
\hline Soil moisture time series & & point & point & 19 & - & half hour instantaneous & $1998-2001$ \\
\hline Soil moisture patterns & & point & point & $5 *$ app. 400 & $0.3-1.4$ & $6-8$ occasions, instantaneous & $1998-2000$ \\
\hline
\end{tabular}

Initial test analyses showed that when excluding nested catchments from the data set, the variograms changed only very slightly, therefore nested catchments were retained in the analysis. Daily precipitation data from 991 stations for the period 1971-1997 were used (Figure 1a). In order to be able to examine spatial aggregation effects, for each day catchment precipitation was calculated by external drift kriging interpolation of the point data for a total of 756 catchments. Catchment precipitation series were again divided into three size classes. In addition, we used one series of precipitation from the Frankenfels precipitation station with hourly data during a period of 7 years to look at the effect of temporal aggregation. Weekly groundwater levels were used. Short records were excluded which left a total of 3539 groundwater stations for the analysis. These data stem from porous aquifers that are mainly located in the valley regions (Figure 1c).

[11] A similarly comprehensive soil moisture data set is not available in Austria. For comparison and to complement the Austrian data, soil moisture data from Australian and New Zealand catchments have been used. The climate in these catchments is not radically different from the Austrian catchments, at least from the lower parts of Austria. The Australian and New Zealand sites have 800-1600 mm mean annual precipitation, 0-600 $\mathrm{mm}$ mean annual runoff depth and potential evapotranspiration on the order of $800-900 \mathrm{~mm}$ per year. Also, hillslope scales do not vary much from the Austrian landscape, so the characteristic scales should be similar within the accuracies of the overall analysis. The soil moisture data stem from five small catchments, Tarrawarra, Satellite Station, Point Nepean, Claydens, and Carrans [Western and Grayson, 1998; Woods et al., 2001]. The sizes of these catchments range from 0.1 to $1.5 \mathrm{~km}^{2}$. The soil moisture data consist of separate temporal and spatial data sets of depth-average volumetric soil moisture in the $0-30 \mathrm{~cm}$ layer. The temporal data were sampled at 19 different locations with $30 \mathrm{~min}$ intervals over three years, using Campbell Scientific CS615 sensors. The spatial data have been sampled at between 285 and 589 points in each catchment on a regular grid using Time Domain Reflectometry. These patterns were collected on between six and eight occasions for each catchment. Table 1 summarizes the data used in this paper.

\section{Method}

\subsection{Transformation of Data}

[12] When performing a variogram analysis, it is useful to first transform the data as, ideally, the analysis should be done on a random variable that exhibits little periodicity with a distribution close to normal [e.g., Sokal and Rohlf, 1995]. We therefore examined a number of transformations to render the data more suitable to variogram analysis. For runoff and precipitation, we examined four steps of transformation, each of them being a transformation of the previous one: (1) untransformed data (specific runoff $\left(\mathrm{m}^{3} \mathrm{~s}^{-1} \mathrm{~km}^{-2}\right)$ ), precipitation (mm/day)), (2) logarithms of data series, (3) seasonal effects removed from the above series, (4) mean of each of the time series subtracted from the above (hereafter referred to as case 1), and (5) the above series divided by their standard deviation (hereafter referred to as case 2). The last transformation produces time series with zero mean and unity standard deviation. For groundwater levels, we examined three steps of transformation: (1) untransformed data (groundwater levels in m), (2) mean subtracted from time series to remove the elevation effects, (3) seasonal effects removed from the above series (hereafter referred to as case 1), (4) above series divided by their standard deviation (hereafter referred to as case 2). Again, the last transformation produces time series with zero mean and unity standard deviation. For soil moisture, we examined two steps of transformation: (1) untransformed data (soil moisture content in percent of total volume), (2) mean over 6-8 occasions of each sampling point subtracted from the spatial data to remove spatial organization; (3) seasonal effects removed from the time series (hereafter referred to as case 1), and (4) spatial data (as above) divided by their temporal standard deviation for each sampling point; Time series (as above) divided by their temporal standard deviation (hereafter referred to as case 2). The last transformation produces spatial patterns of soil moisture for which each point has zero temporal mean and unity temporal standard deviation, and time series with zero mean and unity standard deviation.

[13] For precipitation, the logarithmic transformation is not directly possible, as the logarithm of zero is undefined. Two possible alternatives were considered, treating zero precipitation as no data and padding zero values with the smallest measured precipitation $(0.1 \mathrm{~mm} /$ day $)$. We chose the latter option as this option provides information on the timescales of the interstorm periods. Initial test analyses indicated that the absolute gamma values will be higher when zero is treated as no data compared to padding them. This is because there are less pairs in time or space with equal (zero precipitation) values. However, the shape of the variogram did not change much. In the hourly data series we padded zero precipitation with $0.0041 \mathrm{~mm} / \mathrm{h}$ which is 
equivalent to $0.1 \mathrm{~mm} /$ day. Zero runoff occurred only at a few instances and was treated as no data.

[14] The seasonal components of precipitation, runoff and groundwater levels were estimated by a standard method of moving average estimation (MAE) as used, for example, by De Cesare et al. [2002]. First, we calculated average values of each of the time series for each day of the year. These average values were smoothed by using a moving average procedure with a window of 21 days (precipitation and runoff) and 49 days (groundwater levels). The seasonally smoothed mean for a certain day of year was calculated as:

$$
\overline{u s(i)}=\frac{1}{(2 \cdot \operatorname{iavg}+1)} \sum_{j=-i a v g}^{\text {iavg }} \frac{1}{n_{i j}} \sum_{k=0}^{n_{i j}-1} u(i+j+\operatorname{int}(365.25 k))
$$

where $i$ is the day of the year, iavg is the number of days in the past and future used in the moving averaging window, $2 \cdot i a v g+1$ is the averaging window size, $n_{i j}$ is the number of years with a measurement at day $i+j$ of the year, 365.25 is the average number of days in a year, leap-years included, and $k$ is the year.

[15] The seasonal components of the soil moisture time series were obtained by a Fourier fitting procedure. First the average value of each time series was calculated for each day and time of the year. These averages were then fitted by a two-harmonic Fourier curve. A Fourier curve was used in preference to the moving average described above due to additional variability associated with the shorter time series and the strong link between the soil moisture seasonality and seasonality of potential evapotranspiration associated with changing global radiation, which follows a sinusoidal pattern.

[16] Although the variograms change with the different transformations, they are mostly within an order of magnitude. For runoff and precipitation the data processed by the last two transformations exhibit little skewness and the deterministic or periodic parts in time and space have been removed. We have therefore chosen to focus on these two cases (cases 1 and 2) in the rest of this paper. The groundwater level and soil moisture data exhibit only very minor skewness and so the logarithmic transformation was not needed. However, it was deemed appropriate to remove the deterministic or periodic parts in time and space, so again we focused on cases 1 and 2 in this paper.

[17] The difference between cases 1 and 2 for all data types is that in case 2 the temporal variance is always unity while this is not the case for case 1 . We looked at both cases as for some interpretations it is useful to keep the relative variances in space and time (e.g., for finding space-time pairs of scales) while for other interpretations (e.g., the shape of the variograms) normalization is appropriate.

\subsection{Variograms}

[18] The temporal variograms were calculated as the average of the temporal variograms of the individual time series:

$$
\hat{\gamma}_{t}\left(h_{t}\right)=\frac{1}{\sum_{j=1}^{m} 2 n_{j}\left(h_{t}\right)} \sum_{j=1}^{m} \sum_{i=1}^{n_{j}\left(h_{t}\right)}\left(u\left(\mathbf{x}_{j}, t_{i}+h_{t}\right)-u\left(\mathbf{x}_{j}, t_{i}\right)\right)^{2}
$$

where $h_{t}$ is the time lag, $u\left(\mathbf{x}_{j}, t_{i}\right)$ is the value of the transformed variable at time $t_{i}$ and spatial location $\mathbf{x}_{j}$ of station $j, m$ is the number of data series, and $n_{j}\left(h_{t}\right)$ is the number of pairs with time lag $h_{t}$. For the longest time lags the data were not exhaustively sampled to reduce computation time. Similarly, the spatial variograms were calculated as the average of the spatial variograms of the individual points in time:

$$
\hat{\gamma}_{s}\left(h_{s}\right)=\frac{1}{\sum_{i=1}^{m^{\prime}} 2 n_{i}\left(h_{s}\right)} \sum_{i=1}^{m^{\prime}} \sum_{j=1}^{n_{i}\left(h_{s}\right)}\left(u\left(\mathbf{x}_{j}+\mathbf{h}_{s}, t_{i}\right)-u\left(\mathbf{x}_{j}, t_{i}\right)\right)^{2}
$$

where $h_{s}=\left|\mathbf{h}_{s}\right|$ is the space lag, $u\left(\mathbf{x}_{i}, t_{i}\right)$ is the value of the transformed variable at time $t_{i}$ and spatial location $\mathbf{x}_{j}$ of station $j, m^{\prime}$ is the number of time steps, and $n_{i}\left(h_{s}\right)$ is the number of pairs with space lag $h_{s}$.

[19] To each variogram we fitted visually a variogram model with four parameters. The model is a combination of a fractal model and a Weibull type model:

$$
\gamma(h)=a h^{b}\left(1-\exp \left(-(h / c)^{d}\right)\right)
$$

where $h$ is lag (in either space or time) and $a, b, c$ and $d$ are the parameters. On a double logarithmic plot this variogram model consists of two straight lines with a curve in between. It is therefore possible to determine separately the slopes of the variogram for short lags and long lags. The slope at short lags is $b+d$, while the slope at long lags is $b$. Both slopes can be considered as a fractal power of the variogram but for different lag ranges. The exponential part reaches a sill after a lag dependent on $c$, so $c$ is a parameter controlling the space (or time) scale. $a$ controls the overall level (or variance) of the variogram.

[20] For stationary or approximately stationary variograms (where $b$ is close to zero) a characteristic length or timescale can be defined. One common possibility in geostatistics is to use the range, usually taken as the lag where the variogram reaches $95 \%$ of its maximum value [Webster and Oliver, 2002]. An alternative is to use the $e$-folding distance in space or time. The $e$-folding distance is defined as the distance (in space or time) where the variogram value is $1-1 / e$ of its maximum value. $e$ is the Eulerian constant (2.718). For an exponential variogram $(b=0$ and $d=1)$ in equation (7) the $e$-folding distance is one third of the range. If the variogram is not stationary it is still possible to calculate the range and the $e$-folding distance from the data, but both will depend on the maximum lag over which the data have been sampled. As the estimates of the range are much more sensitive to the fitting procedure than those of the $e$-folding distance, particularly if the variograms are not strictly stationary, in this paper we chose the $e$-folding distance as a measure of the characteristic space scales and timescales. We estimated the $e$-folding distance as

$$
\gamma(e \text {-fold })=\left(1-\frac{1}{e}\right) \sigma^{2}
$$

where $\gamma$ is the fitted variogram model in equation (7) and $\sigma^{2}$ is the sample variance in either space or time. The sample variance in time was found as the average variance of each of the time series. The sample variance in space was found as the average spatial variance for each time step.

[21] Some of the parameters of the variograms are expected to be associated with significant uncertainty even 
though for most instances the sample size is relatively large. Specifically, if either the small-scale fractal power, $b+d$, or the large-scale fractal power, $b$, are estimated from a short range of lags, one would expect them to be poorly defined [see, e.g., Gallant et al., 1994; Katsev and L'Heureux, 2003]. If the fitted variograms are not extrapolated much beyond the range supported by the data, a lack of identifiability will not result in significantly different variograms as alternative models are similar over that range, but it is still very useful to quantify the uncertainties associated with the estimated parameter values, particular if one is to interpret the estimated parameters in terms of their scaling behavior. We therefore performed a split sample analysis and compared variograms of independent subsets of the data. For each catchment size class of runoff, we split the entire data set of 218 catchments (Table 1) into five non overlapping groups of about 44 catchments. In a similar way we split the time series of catchment precipitation, point precipitation and groundwater levels into five groups of approximately equal size. It was not possible to perform the split sample analysis for soil moisture and the hourly rainfall data because of the limited sample size. Variogram models (equation (7)) were then fitted to the sample variograms of each group by an automatic fitting procedure based on weighted least squares of the logarithmic variograms. This procedure resulted in five estimates of each parameter value in each catchment size class. We calculated the variance of each parameter value between the five groups, $\sigma_{b}^{2}$. If the five groups produced similar values of a particular parameter then $\sigma_{b}^{2}$ was small and hence the uncertainty of that parameter was small. Conversely, large $\sigma_{b}^{2}$ indicated large uncertainties. $\sigma_{b}^{2}$ can therefore be considered an estimate of the error variance of the parameter estimate from one group. The error variance associated with the parameter estimates of the entire data set will be smaller than that of each group. We used $\sigma_{e}^{2}$ from equation (9) as a measure of the error variance:

$$
\sigma_{e}^{2}=\frac{1}{m} \sigma_{b}^{2}
$$

where $m$ is the number of groups, i.e., $m=5$. For the sample mean, equation (9) is strictly applicable as the samples are independent. For the parameters of the variogram (equation (7)), the $e$-folding distance and the sample variance equation (9) is an approximation. We examined alternative group numbers which resulted in similar estimates of $\sigma_{e}^{2}$. We therefore believe that equation (9) is a reasonable approximation.

\subsection{Regularization-Spatial Aggregation}

[22] In order to study the spatial aggregation behavior of precipitation and runoff we compared the sample variograms for catchment precipitation and runoff to aggregated or block variograms calculated by regularization from point variograms. The idea of regularization is that the variogram will change its shape when one aggregates or filters the original data. Specifically, the overall level of the variogram (parameter $a$ in equation (7)) will decrease and the $e$-folding distance will increase with increasing aggregation area. Standard procedures for calculating the regularized variograms exist [e.g., Isaaks and Srivastava, 1989]. We used a simple procedure proposed by Sivapalan [1986] and used by Western and Blöschl [1999] and others which builds on the probability density functions (pdf) of the distances within the aggregation area to calculate the aggregated variogram:

$$
\gamma_{\text {agg }}(h, a)=\int_{0}^{r \max } \gamma(r) \cdot f_{2}(r \mid h, a) d r-\int_{0}^{s \max } \gamma(s) \cdot f_{1}(s \mid a) d s
$$

where $h$ is the lag, $a$ is the side length of the aggregation area, $r$ is the distance between two randomly chosen points in each of two aggregation areas $A$ that are separated by a (center to center) distance $h, r_{\max }$ is the maximum of $r, f_{2}$ is the pdf of $r$, $\gamma$ is the point variogram, $s$ is the distance between two randomly chosen points in one aggregation area $A, s_{\max }$ is the maximum of $s, f_{1}$ is the pdf of $s . f_{1}$ and $f_{2}$ are functions of the geometry of the aggregation area. For a square aggregation area, analytical expressions are given by Sivapalan [1986] and Ghosh [1951] and have been used here. Sivapalan [1986] showed that the aggregated variogram is only moderately sensitive to the shape of the aggregation area, so a square shape should also be applicable to natural catchments. The analytical expressions do not cover the case of overlapping squares (i.e., $h<a$ ) for which we evaluated the integral numerically. In calculating the regularized variograms for catchment precipitation and runoff we assumed a side length of the aggregation area $a=\sqrt{A}$ where $A$ is the median area of the catchments in each size class as given in Table 1 . We used point variograms derived from untransformed precipitation data because the aggregation of precipitation by catchments is consistent with mass conservation, a requirement that is fulfilled by aggregating the untransformed data but not fulfilled by the logarithmic data. The regularized variograms were then compared with variograms directly calculated from (untransformed) catchment precipitation for each of the size classes.

[23] For runoff, point time series were not available as the minimum size of gauged catchments in the data set was a few square kilometers. We therefore back calculated a hypothetical point runoff variogram in the following way. We assumed that the point variogram can be represented by equation (7). For a given parameter set we obtained the regularized variograms for the three catchment size classes in a similar fashion as for precipitation. We then optimized the point variogram parameters by fitting the regularized runoff variograms to the respective sample runoff variograms for all three catchment size classes simultaneously. One would expect a simultaneous fit to all three variograms to yield a more robust estimate of the point variogram than a fit to a single variogram. The objective function we minimized consisted of a weighted sum of the squared differences of the logarithms of the sample variograms and the regularized variograms.

\subsection{Space-Time Links}

[24] In order to examine the space-time behavior of characteristic scales we related the spatial variograms to the temporal variograms. Specifically, our main assumption here was to assume that space-time scale pairs characteristic of a process are those scales where the spatial variogram value is equal to the temporal variogram value, i.e.,

$$
\gamma_{t}(T)=\gamma_{s}(L)
$$

where $T$ is timescale, $L$ is space scale, and the $\gamma_{t}$ and $\gamma_{s}$ are the temporal and spatial variograms, respectively. The 
space scale and timescale were then plotted against each other, which gave traces of space-time characteristic scales. If the trace plots as a straight line in a double logarithmic plot, space scale and timescale are related by a power law (equation (3)). The ratio $V=L / T$ is a characteristic velocity, which is a measure of the scales over which measurements become decorrelated from one another. It may be related to a number of physical causes such as the celerity with which a perturbation moves through a system, to particle velocities or to the effects of internal dispersion. Two points separated by a spatial lag $L$ are able to see the same perturbation only if the length of the perturbation is at least $L$. Two points separated by a time $T$ are only able to see the same perturbation if it is moving with a velocity less than $V=L / T$. The correlation in time and space is then related to the possibility that two points separated by a time $T$ or a distance $L$ are able to see the same perturbation. As a natural system consists of a spectrum of small to large perturbations, this possibility will decrease with increasing lags in space and time as the number of perturbations visible to both points is decreasing. The gamma values of the variogram can in a similar way be seen as related to the number of perturbations not visible to both of the points. This applies to both stationary and nonstationary processes. Equating the gamma values in time and space for different gamma values can then be seen as finding the average velocity for a perturbation of size $L$. It is easy to illustrate this for precipitation. A front can be seen as a large-scale perturbation in time and space, while a thunderstorm is a smaller-scale perturbation. The temporal and spatial variograms of precipitation reflect the possibility of seeing these perturbations. Precipitation is also one of the main perturbations for the other processes we have examined here. Additionally, evaporation and snow deposition and melting modulate the dynamics. These perturbations are then filtered by the subprocesses at and under the surface until they reach the catchment outlet where the water flux is actually measured, removing the small-scale components of the perturbations. The variogram still shows the possibility of seeing a perturbation at points separated in time or space, while the velocity found from the method explained above shows the velocities of the filtering processes. Foufoula-Georgiou and Vuruputur [2000] derived space-time pairs of characteristic scales from iso-lines of constant standard deviations for different aggregation levels in space of rainfall fluctuations. This is very similar to equation (11) as the variogram can be interpreted as the lag dependent variance of the fluctuations of a variable. The difference is that FoufoulaGeorgiou and Vuruputur used variance as a function of aggregation area in space and as a function of lag in time while here we examined variance as a function of lag in both space and time.

[25] We compared the spatial and temporal counterparts of the variograms for each data type, e.g., the spatial variograms of runoff from small catchments to the temporal variograms of runoff from small catchments. The exception is hourly precipitation where spatial variograms were unavailable, so we compared the temporal hourly variogram with the spatial variogram from daily point precipitation. The rainfall regime is similar which justifies this comparison. However, because of the differ-
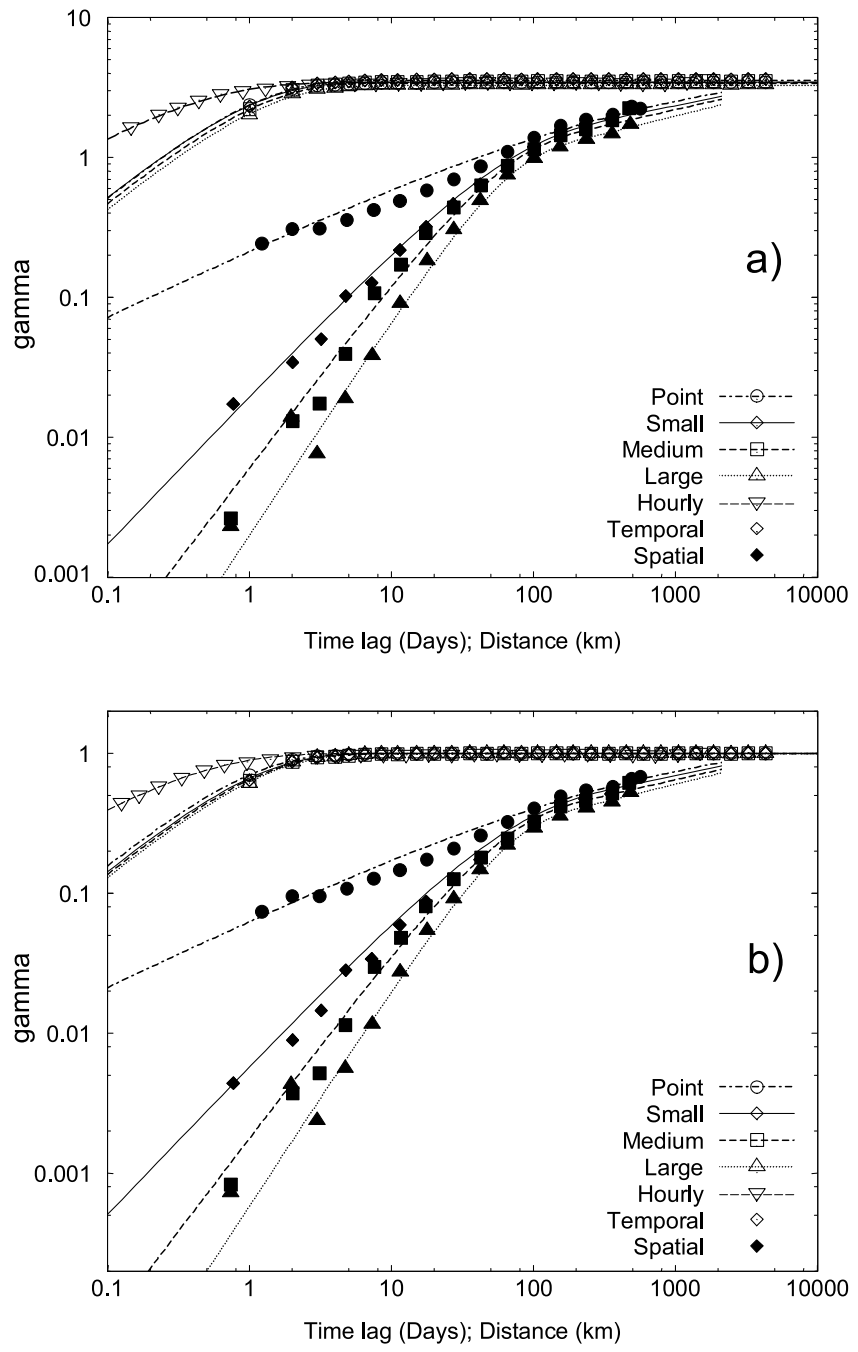

Figure 2. Variograms of precipitation. Spatial sample variograms are shown as solid symbols, and temporal sample variograms are shown as open symbols. Point precipitation is shown as circles, small catchments are shown as diamonds, medium sized catchments are shown as squares, and large catchments are shown as upward pointing triangles. Temporal variogram of hourly precipitation is shown as downward pointing triangles. Fitted variograms are shown as lines: point precipitation is shown as dasheddotted lines, small catchments are shown as solid lines, medium catchments are shown as dashed lines, large catchments are shown as dotted lines, and hourly temporal variogram is shown as a long dashed line. (a) Case 1 and (b) case 2 (see text).

ent time resolutions one would expect some bias to be introduced.

\section{Results}

\subsection{Precipitation}

[26] Spatial and temporal variograms of point precipitation and catchment precipitation for three size classes are shown in Figures 2a (case 1) and 2b (case 2). The temporal variogram for hourly precipitation is also included. The 
Table 2. Parameters of the Fitted Space and Time Variograms as in Equation (7) and Variance for Case $1^{\mathrm{a}}$

\begin{tabular}{|c|c|c|c|c|c|c|c|c|c|}
\hline \multirow[b]{2}{*}{ Process } & \multicolumn{5}{|c|}{ Space } & \multicolumn{4}{|c|}{ Time } \\
\hline & Size & $b+d$ & $b$ & $\sigma^{2}$ & $e$-fold, km & $b+d$ & $b$ & $\sigma^{2}$ & $e$-fold, days \\
\hline Precipitation & point & $0.50,0.01$ & $0.15,0.02$ & $1.67,0.01$ & $47,1.4$ & $0.85,0.01$ & $0.00,0.00$ & $3.41,0.01$ & $0.83,0.00$ \\
\hline Precipitation & small & $1.06,0.06$ & $0.21,0.06$ & $1.39,0.02$ & $59,1.2$ & $0.85,0.00$ & $0.00,0.00$ & $3.64,0.03$ & $0.93,0.01$ \\
\hline Precipitation & medium & $1.33,0.07$ & $0.23,0.02$ & $1.39,0.02$ & $66,1.3$ & $0.85,0.00$ & $0.00,0.00$ & $3.51,0.04$ & $1.00,0.01$ \\
\hline Precipitation & large & $1.54,0.09$ & $0.25,0.06$ & $1.14,0.04$ & $67,3.2$ & $0.85,0.01$ & $0.00,0.00$ & $3.32,0.03$ & $1.04,0.01$ \\
\hline Precipitation & hourly & & & & & 0.65 & 0.00 & 3.25 & 0.3 \\
\hline Runoff & small & $0.58,0.12$ & $0.08,0.04$ & $0.30,0.01$ & $18,3.3$ & $0.61,0.01$ & $0.01,0.01$ & $0.45,0.02$ & $13,1.0$ \\
\hline Runoff & medium & $0.68,0.08$ & $0.08,0.03$ & $0.23,0.02$ & $39,2.8$ & $0.61,0.01$ & $0.01,0.01$ & $0.34,0.02$ & $17,1.0$ \\
\hline Runoff & large & $0.93,0.04$ & $0.08,0.03$ & $0.15,0.00$ & $62,1.9$ & $0.66,0.02$ & $0.01,0.01$ & $0.23,0.01$ & $18,1.2$ \\
\hline Groundwater & & $0.62,0.09$ & $0.12,0.04$ & $0.25 \mathrm{~m}^{2}, 0.01 \mathrm{~m}^{2}$ & $8.3,1.0$ & $0.82,0.01$ & $0.12,0.01$ & $0.29 \mathrm{~m}^{2}, 0.02 \mathrm{~m}^{2}$ & 211,22 \\
\hline Soil moisture & & 0.5 & 0.2 & 6.5 & 0.068 & 0.99 & 0.04 & 1.0 & 9.2 \\
\hline
\end{tabular}

${ }^{\text {a }}$ Here $b+d$ is the fractal power at small scales, $b$ is the fractal power at large scales, $\sigma^{2}$ is the variance in either space or time, $e$-fold is the $e$-folding distance which is a measure of the characteristic scale in either space or time. The nonitalic numbers are the estimates of the parameters, and the italic numbers are the standard deviations $\sigma_{e}$ of the estimation errors of the parameters as of equation (9).

variograms of cases 1 and 2 are very similar. The only significant difference is the overall level of the variograms which is a result of the normalization in case 2 . Tables 2 and 3 (nonitalic numbers) give the fitted variogram parameters $(b, d)$ for cases 1 and 2, respectively, as well as the associated $e$-folding distances and variances. The italic numbers in Tables 2 and 3 are the standard deviations $\sigma_{e}$ of the estimation errors of the parameters.

[27] Figure 2 shows that the temporal variograms are stationary and the $e$-folding distance of all daily variograms (point, small, medium and large catchments) is about one day. This means that very little temporal correlation exists beyond one day. The variogram for hourly precipitation has a significantly shorter $e$-folding distance (only 6 hours). Given that the station used for the hourly variogram has a similar rainfall regime as the daily stations, this difference suggests that the $e$-folding distance from the daily variograms may be biased. The daily sums of precipitation are unable to resolve the shorter timescale fluctuations. On closer examination of the daily variograms for catchments of different sizes (Tables 2 and 3 ) there appears a slight tendency for the $e$-folding distance to increase with catchment size.

[28] In contrast, the spatial variograms for precipitation are all nonstationary. They appear to consist of two fractal parts. At large scales, the fractal power (i.e., the slope in the double logarithmic plot) is on the order of 0.2 ( $b$ in Tables 2 and 3) while at small scales the fractal power varies between 0.5 and $1.55(b+d$ in Tables 2 and 3$)$. There is a clear difference between the spatial variograms of point precipitation and those of catchment precipitation of the different size classes. Point precipitation has the smallest slope at small scales $(b+d=0.5)$ while catchment precipitation for large catchments has the steepest slope at small scales $(b+d=1.55)$. The other catchment size classes are in between. These differences with catchment size translate into differences in the $e$-folding distances which range from about $45 \mathrm{~km}$ (point precipitation) to about $65 \mathrm{~km}$ (catchment precipitation for large catchments). This difference in the $e$-folding distances is larger than the estimation standard deviation, so one would expect it to be significant. There is also a significant decrease of the spatial variance and hence the overall level of the variograms with catchment size (Tables 2 and 3). All of these changes of the spatial variograms with catchment size are related to aggregation effects as will be discussed later in this paper in the context of regularization. The temporal variance is significantly larger than the spatial variance (Table 2).

\subsection{Runoff}

[29] Figures 3a (case 1) and 3b (case 2) show spatial and temporal variograms of runoff. Unlike the variograms for precipitation there are some differences between cases 1

Table 3. Parameters of the Fitted Space and Time Variograms as in Equation (7) and Variance for Case $2^{\mathrm{a}}$

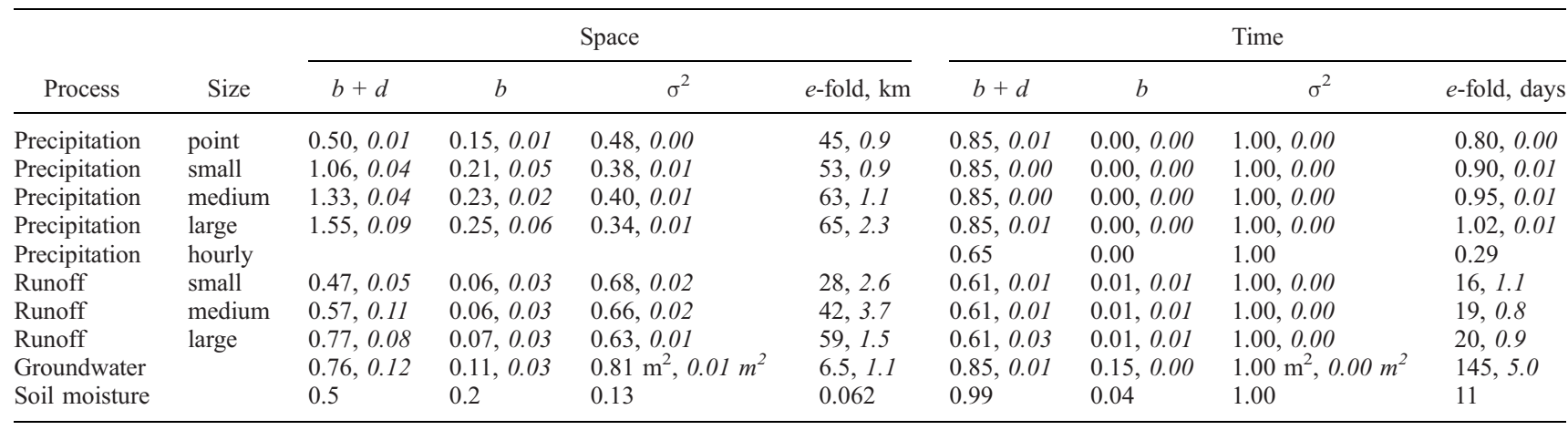

${ }^{\text {a }}$ Here $b+d$ is the fractal power at small scales, $b$ is the fractal power at large scales, $\sigma^{2}$ is the variance in either space or time, $e$-fold is the $e$-folding distance which is a measure of the characteristic scale in either space or time. The nonitalic numbers are the estimates of the parameters, and the italic numbers are the standard deviations $\sigma_{e}$ of the estimation errors of the parameters, as of equation (9). 

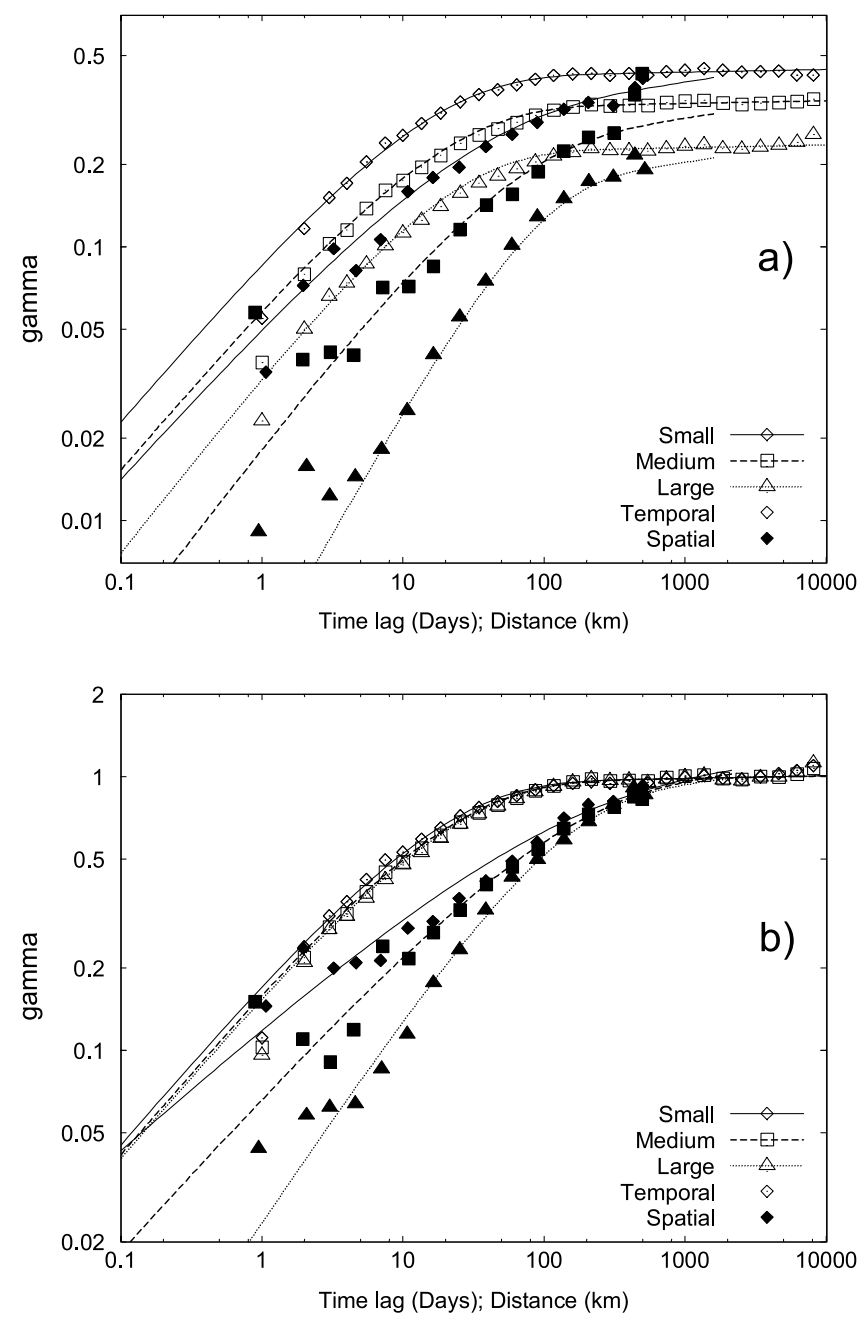

Figure 3. Variograms of runoff. Spatial sample variograms are shown as solid symbols, and temporal sample variograms are shown as open symbols. Small sized catchments are shown as diamonds, medium sized catchments are shown as squares, and large catchments are shown as triangles. Fitted variograms are shown as lines: small catchments are shown as solid lines, medium catchments are shown as dashed lines, and large catchments are shown as dotted lines. (a) Case 1 and (b) case 2 (see text).

and 2, although the main difference is the overall level of the variograms which is a result of the normalization in case 2 . The resulting parameters for cases 1 and 2 are given in Tables 2 and 3, respectively.

[30] The temporal variograms for different catchment sizes are almost stationary ( $b$ is very small) and the $e$-folding distances are about two to three weeks. There is a slight tendency for the $e$-folding distance to increase with catchment size which appears to be significant given that the estimation errors are small (Tables 2 and 3 ). This implies that larger catchments tend to respond more slowly to precipitation forcing than smaller catchments. This is certainly consistent with streamflow analyses in various climates [e.g., Melone et al., 2002]. The main difference of the temporal variograms of the different size classes is their overall level or temporal variance. The temporal variance significantly decreases with catchment size, from 0.44 for small catchments to 0.23 for large catchments. This means that the variability of runoff depth strongly decreases with catchment size. Again, this is consistent with common experience as extreme events (floods and droughts) certainly tend to become more moderate with catchment size if measured in terms of runoff depths or runoff per unit catchment area.

[31] The spatial variograms (Figures $3 \mathrm{a}$ and 3b) for runoff are all nonstationary and appear to consist of two fractal parts although the large-scale part is not as well defined as with precipitation (Figure 2). At large scales the fractal power is about 0.07 ( $b$ in Tables 2 and 3). The associated estimation errors are relatively large (about 0.03). At small scales the fractal power varies between about 0.6 and 0.9 (case 1) and 0.5 and 0.8 (case 2) $(b+d$ in Tables 2 and 3) and is better defined than at the large scales. There is a clear difference between the spatial variograms for the catchments of the different size classes with the larger catchments exhibiting larger fractal power or steeper slopes at small scales than the smaller catchments. These differences with catchment size translate into significant differences in the $e$-folding distance which range from about $20 \mathrm{~km}$ (small catchments) to about $60 \mathrm{~km}$ (large catchments). These differences in the $e$-folding distances are larger than those for catchment precipitation where the $e$-folding distance increased by about $20 \%$ when moving from small to large catchments while the $e$-folding distance tripled in the case of runoff. These changes with catchment size are clearly related to aggregation effects. It appears, however, that catchment runoff exhibits a stronger aggregation behavior than catchment precipitation. This will be discussed later in this paper in the context of regularization. Similarly to the temporal variograms, the overall level of the spatial variograms or spatial variance decreases significantly with catchment size, from 0.30 for small catchments to 0.15 for large catchments (case 1) which is, again, related to aggregation.

[32] The temporal variance is larger than the spatial variance (Table 2) but in comparison with precipitation the difference is smaller. It is also interesting that the spatial variogram of runoff varies by one and a half orders of magnitude (from 0.05 to 1 in Figure $3 b$ ) while the spatial variogram of catchment precipitation varies by almost three orders of magnitude (from 0.001 to 1 in Figure 2b). This suggests that the variability of runoff increases less rapidly with spatial scale than the variability of catchment precipitation which is consistent with the smaller fractal powers of runoff.

\subsection{Groundwater Levels}

[33] Figures 4a (case 1) and 4b (case 2) show spatial and temporal variograms of groundwater levels and the resulting parameters are given in Tables 2 and 3. The two cases are similar with the exception of the fluctuation of the spatial variogram at large space lags for case 1 . These fluctuations are an indication of spatial periodicity but disappear when normalizing the data series (Figure 4b). We believe that this periodicity is related to the geographic position of porous aquifers in Austrian valleys, some of which are regularly spaced (Figure 1).

[34] The temporal variograms are clearly nonstationary and consist of two fractal parts. At large scales, the fractal 

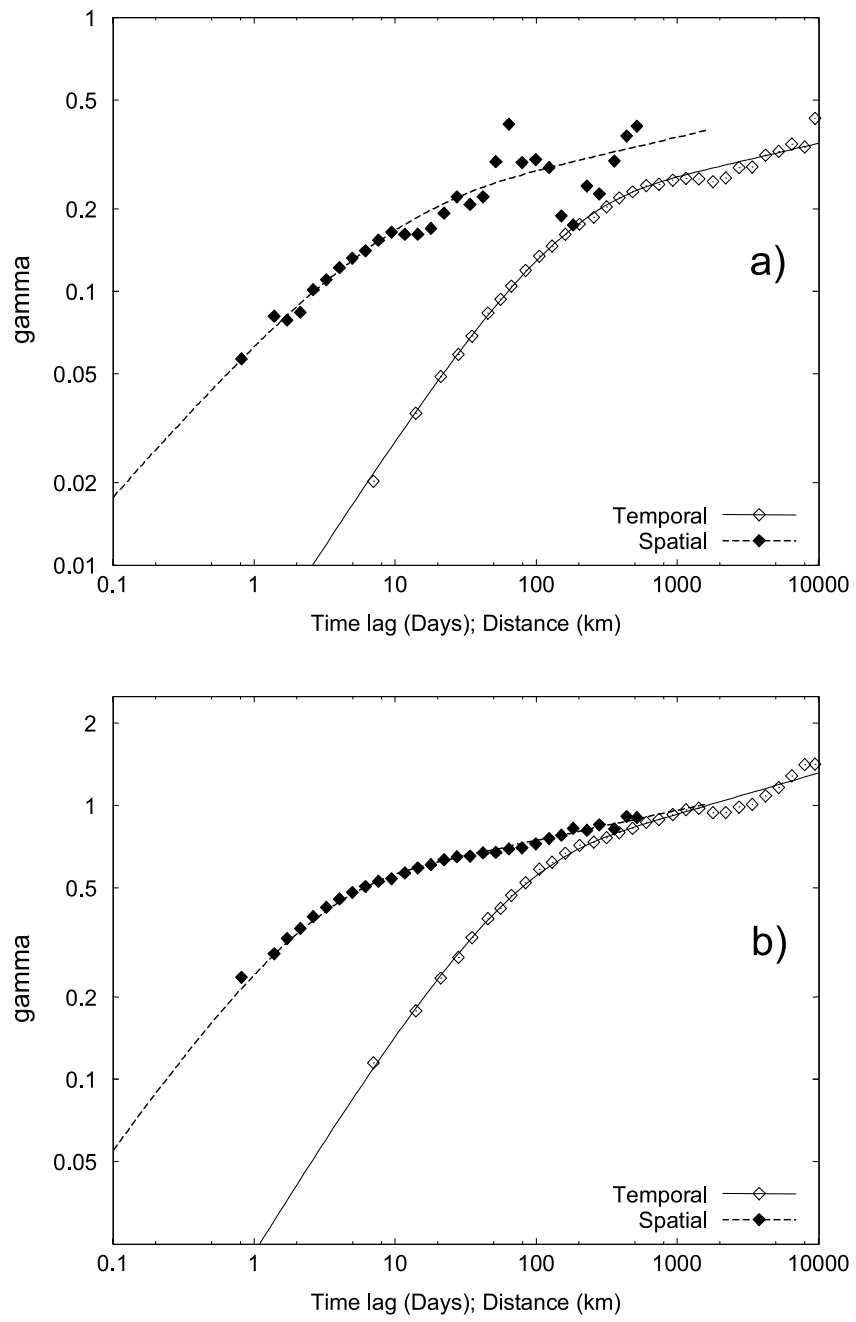

Figure 4. Variograms of groundwater levels. Spatial sample variograms are shown as solid symbols and temporal sample variograms are shown as open symbols. Fitted variograms are shown as lines. (a) Case 1 and (b) case 2 (see text).

power is about 0.1 ( $b$ in Tables 2 and 3) while at small scales the fractal power is about $0.7(b+d$ in Tables 2 and 3 ). The $e$-folding distance in time is on the order of half a year. This very long characteristic timescale is to be expected as groundwater is of course a much more slowly varying process than precipitation and runoff. The temporal nonstationarity is also a reflection of the presence of long timescale fluctuations.

[35] The spatial variograms of groundwater are also nonstationary, as seen in Figure 4. The shapes of the spatial variograms are similar to the shapes of the spatial variograms of catchment precipitation and runoff with two fractal parts. However, the turning point appears to occur at smaller space scales (about $5 \mathrm{~km}$, Figure $4 \mathrm{~b}$ ) than with catchment precipitation and runoff (about $100 \mathrm{~km}$, Figures $2 \mathrm{~b}$ and $3 \mathrm{~b}$ ). This translates into a relatively small $e$-folding distance in space of only about $7 \mathrm{~km}$ in the case of groundwater levels (Tables 2 and 3). This means that groundwater level fluctuations in space are a relatively small-scale process as compared to precipitation and runoff. However, the largescale fractal power is somewhat larger than that of runoff, i.e., groundwater levels are slightly more nonstationary than runoff in space.

[36] The temporal variance is slightly larger than the spatial variance (Table 2) but in comparison with precipitation and runoff the difference is much smaller. This, again, is related to the very slow dynamics of groundwater systems relative to other components of the hydrologic cycle.

\subsection{Soil Moisture}

[37] Figures 5a (case 1) and 5b (case 2) shows spatial and temporal variograms of soil moisture and the resulting parameters are given in Tables 2 and 3. The two cases are similar apart from the overall levels.

[38] The temporal variograms are approximately stationary. The $e$-folding distance in time is on the order of one to two weeks. This is similar to the characteristic timescales found for runoff. This similarity is not surprising as the timescale analysis of runoff tends to mainly capture processes between events, such as moisture redistribution and drainage, as mentioned above, which are also those processes captured by the soil moisture data.
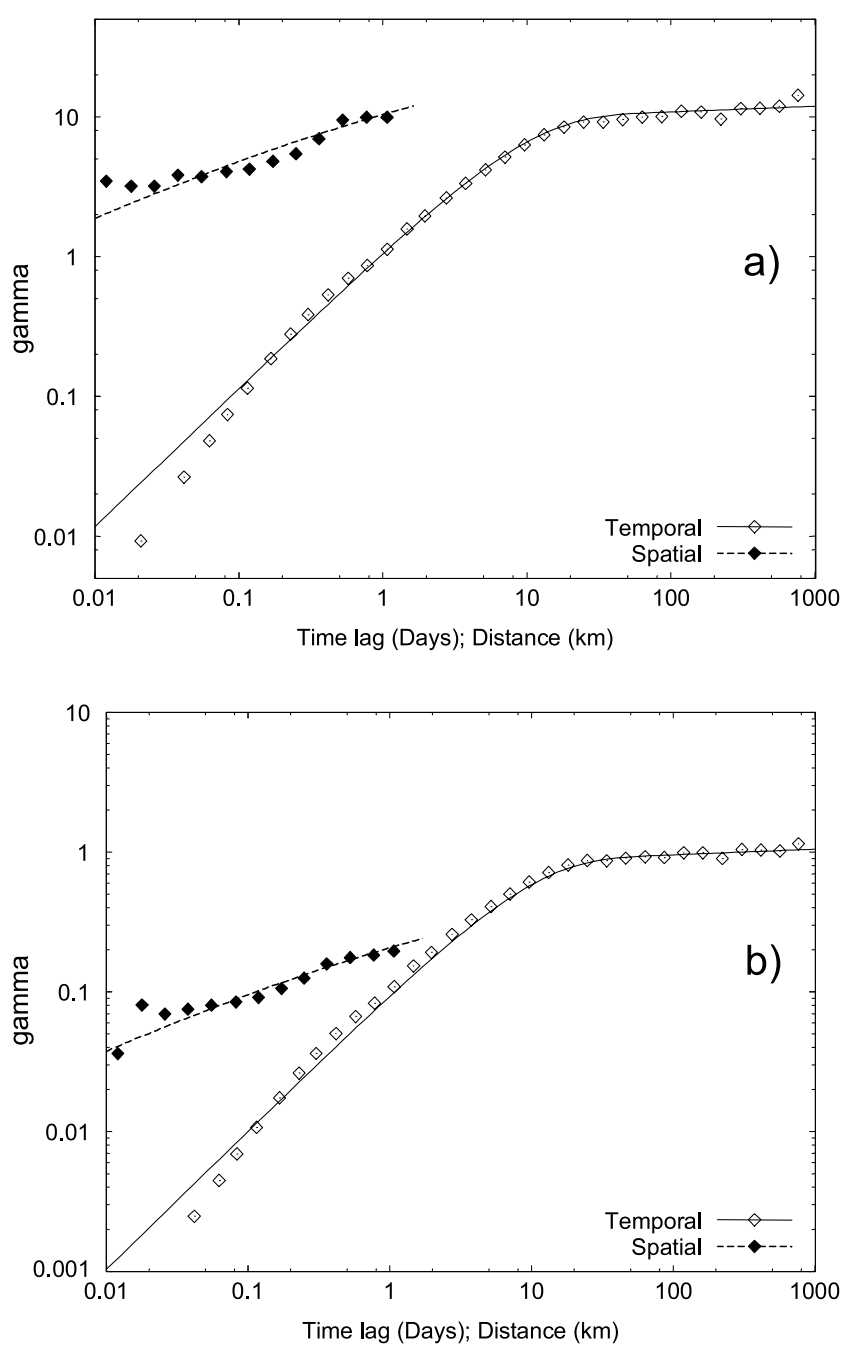

Figure 5. Variograms of soil moisture. Spatial sample variograms are shown as solid symbols, and temporal sample variograms are shown as open symbols. Fitted variograms are shown as lines. (a) Case 1 and (b) case 2 (see text). 
[39] The spatial variograms of soil moisture appear nonstationary; however, the maximum extent does not extend far beyond the hillslope scale and there is little increase in variance for the three points with the largest separations. This suggests that there is significant uncertainty about how the variability might change at larger scales. The moisture patterns appear to exhibit a single fractal behavior without a characteristic scale, although the fit is not very well defined by the data points. It should be noted that random measurement error is about equivalent to $\gamma=2$ in the spatial case, and this may artificially increase the spatial variogram for smaller lags. The fractal power (i.e., the slope in the double logarithmic plot) is on the order of $0.5(b+d$ in Tables 2 and 3). The slope at larger scales $(b)$ is associated with some uncertainty as it has been extrapolated beyond the data points. The spatial variograms cover only two orders of magnitude in space, which is significantly less than for the other variables examined here. The temporal variance is slightly larger than the spatial variance (Table 2), as is the case for groundwater levels, although they are not strictly comparable because of the different extents over which the data have been collected (Table 1).

\subsection{Regularization-Spatial Aggregation}

[40] The spatial aggregation behavior of precipitation and runoff has been analyzed in more detail by making comparisons with regularized variograms. Figure 6a shows the results for the case of precipitation. The solid circles are the spatial variogram of point precipitation similar to that in Figure 2 but based on untransformed data. To this data-derived variogram we fitted a variogram model shown as a dashed-dotted line in Figure 6a. We then applied the regularization procedure (equation (10)) to this fitted point variogram to calculate aggregated or block variograms. The size of the aggregation area ( $a$ in equation (10)) used was $6,11.6$ and $28.6 \mathrm{~km}$ for small, medium and large catchments, respectively, which are the square roots of the median catchment sizes as shown in Table 1. The three aggregated variograms so estimated are shown as solid, dashed and dotted lines in Figure 6a. For comparison, the variograms that have been directly estimated from catchment precipitation are shown as symbols. The solid diamonds, squares and triangles are the spatial variograms of catchment precipitation for small, medium and large catchment classes, respectively, all based on untransformed data. As can be seen from Figure 6a, the regularized variograms fit closely to their counterparts directly derived from catchment precipitation. This suggests that precipitation aggregates linearly in space and can be represented by simple aggregation concepts as would be expected.

[41] Figure $6 \mathrm{~b}$ shows the results for the case of runoff, all based on untransformed data. As point runoff data were not available we back calculated a hypothetical point runoff variogram by fitting the regularized runoff variograms to the sample variograms of the three catchment size classes as described in section 3.3. The three sample variograms are shown as solid diamonds, squares and triangles and the three regularized variograms fitted to them are shown as solid, dashed and dotted lines, corresponding to small, medium and large catchment classes, respectively. Figure $6 \mathrm{~b}$ suggests that the regularized variograms provide a reasonable and
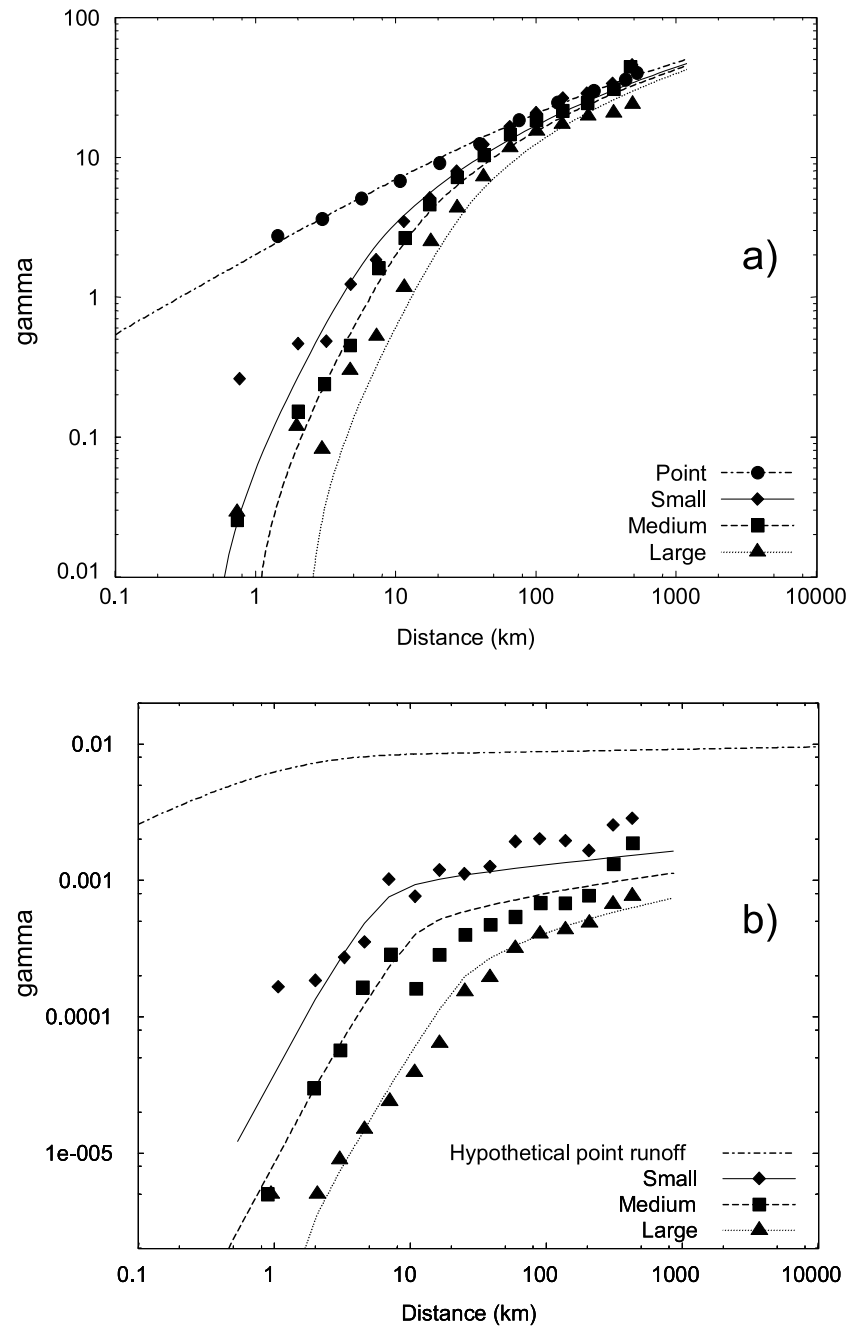

Figure 6. Comparison of regularized spatial variograms with sample variograms for (a) precipitation (untransformed daily precipitation) and (b) runoff. In Figure $6 a$ the dasheddotted line is a fit to the point sample variograms (circles) of precipitation. The other lines have been derived from regularization and fit the sample variograms of catchment precipitation (symbols) well. In Figure $6 \mathrm{~b}$ the dashed-dotted line relates to hypothetical point runoff and has been back calculated by fitting the regularized catchment variograms (other lines) to the catchment sample variograms (symbols).

consistent fit to all three sample variograms. It is now interesting to examine the back calculated variogram of hypothetical point runoff shown as a dashed-dotted line in Figure $6 \mathrm{~b}$. The variogram parameters (equation (7)) of this point variogram are $b+d=0.58$ and $b=0.017$, and the variance and the $e$-folding distance are about $\sigma^{2}=0.009$ $\mathrm{mm}^{2} \mathrm{~d}^{-2}$ and $e$-fold $=0.7 \mathrm{~km}$, respectively. This implies that hypothetical point runoff varies over very short space scales, much shorter than catchment runoff, and that its variance is much higher than that of catchment runoff $\left(\sigma^{2}=\right.$ $0.00190,0.00085$, and $0.00048 \mathrm{~mm}^{2} \mathrm{~d}^{-2}$ for the three catchment size classes of untransformed data in Figure $6 b)$. The point variogram for runoff is much flatter than that for precipitation and the decrease of variance (as represented by the overall level of the variograms in Figure 


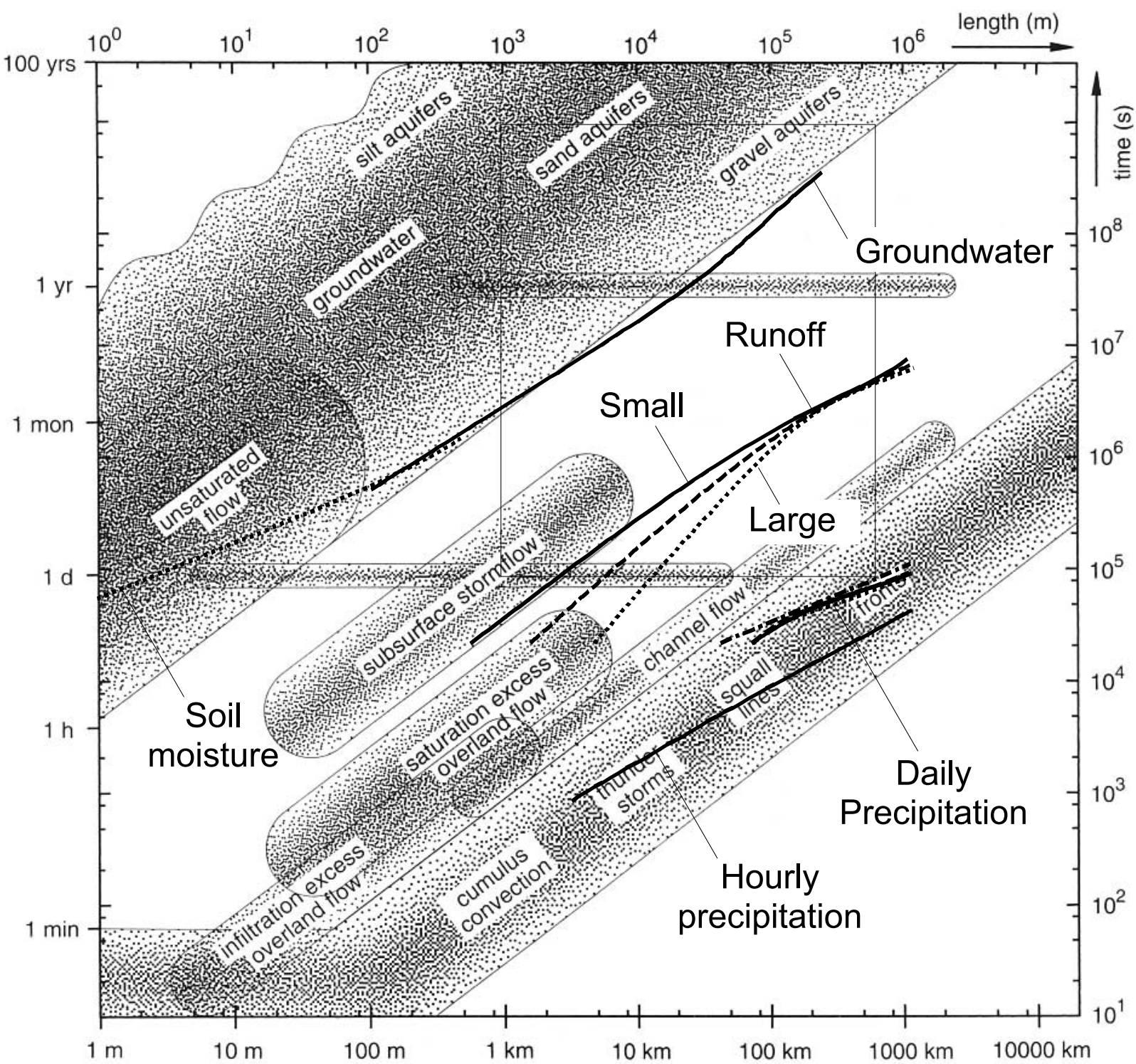

Figure 7. Schematic relationship between spatial and temporal process scales in hydrology [from Blöschl and Sivapalan, 1995] shown together with the space-time traces of characteristic scales obtained in this paper. The rectangle indicates the minimum spacing and the maximum extent of the precipitation and runoff data, both in space and time.

6b) with catchment size is larger than for the case of precipitation (Figure 6a).

\subsection{Links Between Space Scales and Timescales}

[42] The characteristic space-time scales have been derived as those scale pairs where the spatial variogram value is equal to the temporal variogram value (equation (11)). The variograms for case 1 for each of the variables considered have been used (Figures 2a, 3a, 4a, and 5a), as the relative variances in space and time should be reflected in the scale pairs, so normalization by the variance is not appropriate. The space scale-timescale traces are shown in Figure 7. Each line shows the trace for one of the data types representing one process. The traces have been slightly extrapolated beyond the range covered by the data points
(Figures 2a, 3a, 4a, and 5a) but only to an extent we deemed reliable given the fit of the variogram models to the variograms estimated from the data. The box in Figure 7 shows the minimum spacing and the maximum extent of the available precipitation and runoff data, both in space and time, i.e., $1 \mathrm{~km}$ to $700 \mathrm{~km}$ and 1 day to 30 years. The box thus represents the space-time range over which the precipitation and runoff data are reliable without extrapolation. As all of the precipitation data types lie outside the box, the precipitation space-time scales obtained here need to be interpreted with caution. However, the runoff traces are well within the reliable range. The traces in Figure 7 have been superimposed on the figure taken from Blöschl and Sivapalan [1995] representing schematic relationships between spatial and temporal process scales in hydrology. 
Table 4. Characteristic Velocities and Scaling Exponents $z$ in Equation (3) for Case $1^{\text {a }}$

\begin{tabular}{|c|c|c|c|c|c|c|c|c|}
\hline Process & Size & $0.01 \mathrm{~km}$ & $0.1 \mathrm{~km}$ & $1 \mathrm{~km}$ & $10 \mathrm{~km}$ & $100 \mathrm{~km}$ & $1000 \mathrm{~km}$ & z \\
\hline Precipitation & point & & & & & (3.1) & (10) & 0.5 \\
\hline Precipitation & small & & & & & (3.7) & (12) & 0.5 \\
\hline Precipitation & medium & & & & & (3.6) & (12) & 0.5 \\
\hline Precipitation & large & & & & & (3.9) & (13) & 0.5 \\
\hline Precipitation & hourly & & & & $(5.5)$ & 12 & 27 & 0.6 \\
\hline Runoff & small & & & $(0.032)$ & 0.040 & 0.069 & $(0.18)$ & 0.8 \\
\hline Runoff & medium & & & $(0.089)$ & 0.075 & 0.082 & $(0.20)$ & 1.0 \\
\hline Runoff & large & & & & $(0.190)$ & 0.096 & $(0.19)$ & 1.3 \\
\hline Groundwater level (velocities $\times 10^{-3}$ ) & & & & 0.39 & 0.68 & 0.80 & $(0.75)$ & 0.8 \\
\hline Soil moisture (velocities $\times 10^{-3}$ ) & & 0.063 & 0.20 & 0.28 & & & & 0.5 \\
\hline
\end{tabular}

${ }^{\mathrm{a}}$ Numbers in parentheses have been extrapolated beyond the data. Velocities have units of $\mathrm{m} / \mathrm{s}$.

It is now interesting to compare whether the traces obtained from the variogram analysis fit to the schematic. In order to assist with the interpretation two quantities have been derived from the space-time traces. The first is the characteristic velocity $V=L / T$ where $T$ is timescale and $L$ is space scale. The characteristic velocities are a measure of the position of the traces in Figure 7 with high velocities plotting on the bottom right-hand side and low velocities plotting on the top left-hand side. The second quantity is the scaling exponent $z$ as in equation (3). The scaling exponent is equivalent to the slope of the traces in Figure 7 as it is a double logarithmic plot. The scaling exponents have been obtained by fitting equation (3) to the traces over the reliable scale range. The characteristic velocities for different space scales and the scaling exponents are summarized in Table 4. Values shown in brackets have been extrapolated and are less reliable than the other values. For comparison, the same quantities have been derived but for transformation case 2 to examine the effect of the transformation (Table 5). Most of the values are very similar although groundwater and soil moisture characteristic velocities are somewhat larger for case 2. This is related to the relative magnitudes of the spatial and temporal variances which for these two variables do change when normalized (Figures 4 and 5) while they do not for precipitation and runoff (Figures 2 and 3 ).

[43] For precipitation the traces of space-time scales in Figure 7 fall within those shown as fronts and squall lines in the schematic. There seems to be little difference between the traces for point precipitation and the traces for catchment precipitation for catchments of different size classes. For daily precipitation the characteristic velocities are of the order of 3 to $10 \mathrm{~m} / \mathrm{s}$, which is slightly lower than what one would expect from the literature. There is likely some bias due to using daily precipitation sums, which can easily account for these differences. The trace for hourly precipitation has larger characteristic velocities than that of daily precipitation (about 5 to $30 \mathrm{~m} / \mathrm{s}$ ). Even though these traces need to be treated with caution as they stem from a combination of temporal hourly data and spatial daily data they seem to be less biased when compared with the schematic. The slopes or scaling exponents of the traces of precipitation are all about 0.5 (0.6-0.7 for hourly precipitation), which is lower than the 0.9 consistent with the schematic. This implies that the characteristic velocities increase significantly with scale. This might also be due to the use of daily precipitation data because the greatest smoothing occurs at the shortest timescales, which is likely to result in a greater underestimation of velocities for the short timescales and thus a lower scaling exponent.

[44] For runoff the space scale-timescale traces are slower than the scales shown for channel flow in the schematic and larger than the scales shown for subsurface storm flow and saturation excess overland flow. As suggested by Blöschl and Sivapalan [1995], their schematic shows individual processes. Runoff, however, is the combined result of runoff generation processes such as subsurface storm flow and saturation excess overland flow and runoff routing in the stream channel. The combined process will likely have the largest of the timescales of the component processes because runoff can be thought of as a system in series consisting of runoff generation and routing. Also, the runoff generation processes shown in the schematic are mainly related to individual events while the temporal variograms have been derived from continuous runoff data. This means that the variograms will also capture slower processes between events, such as moisture redistribution and drainage, and hence exhibit somewhat slower scales than shown for the events in the schematic. The characteristic velocities for

Table 5. Characteristic Velocities and Scaling Exponents $z$ in Equation (3) for case $2^{\mathrm{a}}$

\begin{tabular}{|c|c|c|c|c|c|c|c|c|}
\hline Process & Size & $0.01 \mathrm{~km}$ & $0.1 \mathrm{~km}$ & $1 \mathrm{~km}$ & $10 \mathrm{~km}$ & $100 \mathrm{~km}$ & $1000 \mathrm{~km}$ & $z$ \\
\hline Precipitation & point & & & & & $(3.2)$ & $(10)$ & 0.5 \\
\hline Precipitation & small & & & & & $(3.4)$ & (11) & 0.5 \\
\hline Precipitation & medium & & & & & $(3.5)$ & (12) & 0.5 \\
\hline Precipitation & large & & & & & $(3.9)$ & (13) & 0.5 \\
\hline Precipitation & hourly & & & & $(5.3)$ & 11 & 25 & 0.7 \\
\hline Runoff & small & & & $(0.023)$ & 0.041 & 0.072 & $(0.030)$ & 0.8 \\
\hline Runoff & medium & & & $(0.055)$ & 0.066 & 0.081 & $(0.061)$ & 0.9 \\
\hline Runoff & large & & & & $(0.165)$ & 0.100 & $(0.089)$ & 1.2 \\
\hline Groundwater level (velocities $\times 10^{-3}$ ) & & & & 0.56 & 1.1 & 4.3 & $(9.4)$ & 0.8 \\
\hline Soil moisture $\left(\right.$ velocities $\left.\times 10^{-3}\right)$ & & 0.31 & 1.2 & 4.9 & & & & 0.4 \\
\hline
\end{tabular}

${ }^{a}$ Numbers in parentheses have been extrapolated beyond the data. Velocities have units of $\mathrm{m} / \mathrm{s}$. 
runoff are on the order of $0.04-0.2 \mathrm{~m} / \mathrm{s}$ which is slower than what one would expect for channel flow $(1 \mathrm{~m} / \mathrm{s})$ and similar to what one would expect for subsurface storm flow and saturation overland flow. The traces for catchments of different size classes are significantly different in terms of their slope. The smallest catchments have the smallest scaling exponent $(0.8)$, indicating increasing velocities with scale, while the largest catchments have the largest scaling exponent (1.3), indicating decreasing velocities with scale. The differences between catchment sizes are much larger for runoff than they are for precipitation.

[45] For groundwater levels, the space scale-timescale traces are close to the scales shown for gravel aquifers in the schematic. As would be expected the characteristic velocities are much slower than those for the other processes and are on the order of $5 \times 10^{-4} \mathrm{~m} / \mathrm{s}$ which is about $50 \mathrm{~m} /$ day. This is faster than what one would expect as an average flow velocity in all the aquifers from which the data have been taken. It is likely that the characteristic velocities here are related to the celerity with which a perturbation moves through a system. Pressure waves in groundwater systems move much (10 to 100 times) faster than flow velocities, particularly in confined aquifers, which explains the difference. The slope or scaling exponent of the trace of groundwater levels is about 0.8 , which is slightly lower than the 0.9 consistent with the schematic. This implies that the characteristic velocities increase slightly with scale.

[46] For soil moisture, the space scale-timescale trace plots directly on those shown for unsaturated flow in the schematic. The characteristic velocities range between $1 \times$ $10^{-4}$ and $3 \times 10^{-4} \mathrm{~m} / \mathrm{s}$ which are about 0.3 to $1 \mathrm{~m} /$ hour. The slope or scaling exponent of the trace is about 0.5 , which implies that the characteristic velocities increase significantly with scale.

\section{Discussion and Conclusions}

\subsection{Timescales}

[47] Characteristic timescales exist for both precipitation and runoff as the temporal variograms are stationary. Their $e$-folding distances are on the order of 1 day and two to three weeks, respectively. Soil moisture is only approximately stationary with a characteristic timescale of about two weeks. As we move down into the subsurface the temporal variograms become even more nonstationary and the $e$-folding distance increases. The groundwater level variograms exhibit an $e$-folding distance of about half a year. This increase in the characteristic timescales can be thought of as a filtering effect as the water moves on the surface and into the subsurface which removes some of the short term fluctuations and imposes a longer memory on the time variations. This filtering, if linear, can be mathematically represented by a convolution operation of routing, both on the surface and in the subsurface, which will always increase the characteristic scale [e.g., Dooge, 1973]. Even if most of the hydrologic processes involved here are known to be nonlinear, qualitatively, linear system theory provides a nice framework for the increase in timescales as water flows through a series of compartments.

[48] These filtering concepts can also be used to illustrate the increase in nonstationarity as one moves down into the subsurface. The fractal power of the variogram $(b)$ can be seen as the ratio of large-scale variability and small-scale variability [e.g., Blöschl, 1996]. While the small-scale variability of precipitation in time is very high, and practically removes our ability to see the long time variability $(b=0)$, some of the small-scale variability is filtered out for the other processes. For runoff, $b$ is very small but greater than zero, for soil moisture $b$ is about 0.04 , and for groundwater levels $b=0.1$. The slower processes are more affected by the long time variability of precipitation. Indeed, if one fits equation (7) to a variogram estimated from time series of annual precipitation totals (not shown here), $b$ is about $b=0.1$ for the Austrian data used here. This similarity with the long term behavior of groundwater levels is quite remarkable.

[49] It is interesting to relate these results to the huge body of hydrological literature on time series analysis [see, e.g., Fleming et al., 2002]. For many variables, short records will indicate stationary behavior although, as the record length increases, there is evidence for nonstationarity [e.g., Kirchner et al., 2000], particularly if one examines extremes as in the classical paper of Hurst [1951]. The stationary behavior of precipitation is also partly due to our choice of examining continuous time series of precipitation. If the time variability within events is examined, most analyses find nonstationary behavior, which can be well explained by different fractal concepts [e.g., Seed et al., 1999].

[50] The effect of catchment size on the temporal $e$-folding distance of both precipitation and runoff is small. For precipitation there is a slight tendency for temporal $e$-folding distances to increase with catchment size ( 0.83 days for points, 1.04 days for large catchments). We used daily data and it is possible that the effect would be larger if we used higher resolution precipitation data. The precipitation analyses of Seed et al. [1999], for example, showed that the temporal $e$-folding distances for big grid elements $(128 \times$ $128 \mathrm{~km}$ ) were significantly longer than for small grid elements $(4 \times 4 \mathrm{~km})$ although their work focused on individual storms rather than continuous series. While the overall levels of the variograms (i.e., the variances) of runoff decrease dramatically with catchment size the increase in the temporal $e$-folding distance is relatively small. Apparently, the tendency of slower response times with increasing catchment area that one would expect, only slightly changes the relatively constant temporal variability of the precipitation forcing, as precipitation becomes runoff. This implies that the slowest process (i.e., that which controls the extent of temporal smoothing of precipitation) is related to generation of runoff rather than routing of runoff. This is supported by the similarity in $e$-folding timescales for runoff and soil moisture. It is likely that this behavior would change when one moves to the very big catchments around the world in which routing times of months occur.

\subsection{Space Scales}

[51] Characteristic space scales do not exist for point precipitation as the spatial variograms are clearly nonstationary and approximately fractal. This is consistent with a substantial body of literature on the fractal behavior of rainfall processes [e.g., Lovejoy and Schertzer, 1985] although some authors state that spatial rainfall variability 
may exhibit a more complex behavior than that suggested by the power law in equation (1), e.g., multifractality [Gupta and Waymire, 1990]. As one moves from point precipitation to catchment precipitation, a break appears in the variograms which is more pronounced for large catchments than it is for small catchments. Clearly, the averaging over a catchment area imposes a characteristic spatial scale on precipitation. These aggregation effects are consistent with spatial regularization methods, i.e., simple linear aggregation or filtering in space. The main effect of the aggregation by catchments is to filter out the small-scale variability (i.e., the variograms get steeper at short lags), to reduce the overall variance (i.e., the overall level of the variograms decreases), and to increase the $e$-folding distance. The spatial variograms of runoff are similar to those of catchment precipitation in that they exhibit a break, and consist of a steep part at short lags and a flatter part at large lags. Again, catchment size appears to impose a characteristic spatial scale. When moving from small catchments to large catchments the characteristic scales of runoff increase from about $20 \mathrm{~km}$ to about $60 \mathrm{~km}$. The characteristic space scale of back calculated point runoff is much smaller $(0.7 \mathrm{~km})$ which is consistent with this catchment scale effect. These differences in the $e$-folding distances are larger than those for catchment precipitation where the $e$-folding distance increased by about $20 \%$ when moving from small to large catchments while the $e$-folding distance tripled in the case of runoff.

[52] Catchment runoff exhibits a stronger aggregation behavior than catchment precipitation. Woods et al. [1995] performed a similar analysis of spatial variance of runoff although their catchment sizes were smaller $\left(<10 \mathrm{~km}^{2}\right)$ than in this paper. They found that the variance between catchments decreased faster than with the inverse of catchment area. A decrease of variance with the inverse of catchment area would result if point runoff were an uncorrelated random field. They interpreted the faster decrease as an evidence of spatial organization. An alternative interpretation is that their fast decrease is a result of increasing catchment response times with catchment scales, i.e., space-time aggregation effects. In this paper the spatial variance of runoff does decrease strongly with catchment size, but it is less than with the inverse of catchment area (e.g., $\sigma^{2}=0.3$ for a medium catchment size of $36 \mathrm{~km}^{2}$ and $\sigma^{2}=0.15$ for a medium catchment size of $701 \mathrm{~km}^{2}$, Table 2). Hypothetical point runoff may therefore be spatially correlated which is consistent with the results from the regularization analysis.

[53] It is also of interest to compare the spatial variograms of runoff obtained here with the theoretical variograms of Gottschalk [1993]. The main difference between the variograms of Gottschalk and those derived here is that Gottschalk [1993] took into account the spatial network structure while this has not been done here. He suggested that the organization of the landscape into catchments implies that the spatial runoff field is nonhomogeneous and can be better described by a tree-structure than by a homogeneous random field. With this type of spatial structure, the variograms are no longer second order stationary but will change with location. Gottschalk [1993] assumed an exponential spatial variogram for hypothetical point runoff applicable to the average runoff from a number of events. He then plotted the correlation against distance along the river rather than lag as has been done here. When going from points to catchments the shape of the variograms changed in a similar fashion as it does in this paper. The overall level of the variograms decreased (i.e., better correlations) and the spatial $e$-folding distances increased (i.e., correlations over longer distances) as catchment size increased. It would be interesting to apply the method of Gottschalk [1993] to the data used here to see how much the effects of nonhomogeneity will change the spatial variogram shapes.

[54] As one moves from catchment precipitation to runoff, the spatial $e$-folding distance decreases for the small catchments and it is still shorter for groundwater levels $(59 \mathrm{~km}, 18 \mathrm{~km}$ and $8 \mathrm{~km}$ for catchment precipitation, runoff and groundwater levels, respectively, Table 2). This means that the characteristic spatial scales of the processes decrease as the water moves through the hydrologic system. We suggest that this is the result of a superposition of the small-scale variability of catchment and aquifer properties on the rainfall forcing. Both soils and aquifer characteristics tend to by highly variable at small scales [see Roth et al., 1990; Gelhar, 1993, p. 292]. These smaller scales are then imposed on the spatial variability of the water fluxes.

[55] It is interesting to contrast this reduction of spatial scales with the increase in temporal scales by catchments and aquifers discussed above. The main reason for this difference is that, in the time domain, the routing processes can be thought of as a convolution which will always increase the characteristic scales as the water flows on the surface and through the subsurface. This aggregation in time also effectively results in a smoothing of the precipitation forcing of the system in space as a consequence of the space-time correlations in the precipitation field. However, this space-time smoothing of forcing is dominated by a second spatial effect. The most important effect in the space domain is not a convolution that takes place for a given catchment area but rather it is the transformation of rainfall to rainfall excess and recharge which can be represented, for example, by a multiplicative or additive operation [see, e.g., Woods and Sivapalan, 1999]. In addition to this spatially variable point scale transformation, processes such as lateral flow can add spatial variability at small spatial scales, as is the case with soil moisture on hillslopes. If a large-scale random field (precipitation) is combined with a small-scale random field (soils) the resulting field will exhibit intermediate scales and this is what we see for runoff and groundwater levels. As one moves from small to large catchment runoff the spatial scales increase because of aggregation effects.

[56] The $e$-folding distance we found for soil moisture is much smaller than that for the other processes. This fits into the above reasoning of soil characteristics imposing short space scales. However, it should be noted that the overall extent of the data used here was much smaller than that of the other variables which may bias the estimated characteristic scales toward smaller scales [e.g., Blöschl, 1999]. It is also worth comparing the fractal shape of the spatial variograms found here to reviews of Western et al. [1998, 2002]. Most of the variograms compiled by Western et al. suggest stationary spatial soil moisture variability with characteristic scales on the order of tens to hundreds of meters. It is 
possible that part of the difference between the results of this paper and those of Western et al. [1998, 2002] is related to removing the spatial organization of the soil moisture patterns here, as we focused on the random part of the variability rather than on the deterministic part. The spatial organization imposed by the landscape may exhibit a characteristic spatial scale [Western et al., 1999], which however will not be apparent if only the random component is examined. Also it should be recalled that the shape of our variograms at the largest lags suggests some uncertainty in the interpretation of stationarity. However, where intermediate extents of up to tens of $\mathrm{km}$ have been examined, fractal behavior has been observed [Rodríguez-Iturbe et al., 1995]. This may suggest that the underlying soil moisture variability is indeed nonstationary, which may however not appear in the variogram analysis because of the limited size of an individual case study. It should be noted that soil moisture is a bounded variable, so one would not expect the variogram to increase without bounds. Indeed, Entin et al. [2000] found stationary behavior at scales above hundreds of kilometers.

\subsection{Links Between Space Scales and Timescales}

[57] The space-time traces of characteristic scales as derived from equating the variogram values of the spatial variograms and those of the temporal variograms fit well into the schematic diagram of space-timescales proposed by Blöschl and Sivapalan [1995]. The characteristic velocities for precipitation and runoff are on the order of 10 and $0.1 \mathrm{~m} / \mathrm{s}$ respectively. The characteristic velocities of both groundwater levels and soil moisture range between $10^{-4}$ and $10^{-3} \mathrm{~m} / \mathrm{s}$. There are order of magnitude differences between the different processes, and it is these one means to capture when characterizing processes by characteristic scales as discussed in the introduction. There are some minor differences between the schematic and the traces found here which can all be explained on the basis of process reasoning and data characteristics. The traces for daily precipitation are slightly slower than those in the schematic, which may be related to biases imposed by using daily precipitation data. The traces for hourly precipitation are slightly faster than those in the schematic, which may be due to biases introduced by combining hourly temporal variograms and daily spatial variograms. For example, Seed et al. [1999] showed that hourly precipitation is less well correlated in space (shorter $e$-folding distances) than daily precipitation, so the assumption made here may overestimate the characteristic velocities slightly.

[58] The characteristic velocities of runoff found here are somewhat slower than those suggested by the schematic of Blöschl and Sivapalan [1995]. As suggested earlier in this paper this is likely to be due to both the processes and the scales considered. The schematic focuses on single events while we examined continuous series and the schematic also represents individual processes while we examined runoff as the combination of a number of processes. Our analysis utilized daily flow data and most catchments were less than $1000 \mathrm{~km}^{2}$, thus the routing times for surface runoff would generally be less than a day. However the temporal scales of runoff are much longer than a day. This means that the temporal pattern of flow is dominated by the temporal behavior of precipitation forcing and runoff generation processes at daily and longer timescales, not by the routing of surface runoff, in catchments of this size. Comparisons of the small, medium and large catchments show that neither the scales of the temporal forcing or the filtering of that forcing depend on catchment size. However there is a significant increase in the spatial scales as one moves from small to large catchments. This combined with the spatially independent temporal scales leads to the apparent increase in velocity with spatial scale.

[59] In general there is a fundamental difference in how the landscape modifies the meteorological forcing in space and time. It removes temporal variability and adds spatial variability. The landscape can be conceptualized as a collection of parallel (vertical stores) that interact laterally to some extent via different flow paths, including overland and shallow subsurface flow paths determined by, and thus limited to the scale of, the topography as well as deeper groundwater flows through aquifers, typically at somewhat larger scales. There are also spatial differences in these stores due to variations in soil and vegetation properties. The stores act in time to filter out variance in the forcing, especially at smaller temporal scales.

[60] These storage effects increase the characteristic timescale from about 1 day for rain to 10 days for soil moisture and a hundred days for groundwater, which also becomes nonstationary as a consequence. The runoff response timescales are a mix of the soil moisture and groundwater response timescales because runoff is influenced by both soil moisture and groundwater. The soil moisture affects the partitioning of rainfall into infiltration and runoff, as well as the subsurface storm flow and interflow processes. The groundwater affects the base flow. However spatial differences between the stores mean that this temporal filtering is spatially variable. Milly and Wetherald [2002] showed how storage in the saturated zone and in surface waters acts as a low-pass filter that damps small-scale variability in the time domain, but leaves large-scale variability unmodified from that of runoff production. The strength of the filter, in their paper quantified by an effective water residence time, varied widely from one catchment to another.

[61] In addition there are lateral interactions between these stores. Some of these, such as subsurface lateral flow, also add to the spatial variability of the soil moisture field and hence the runoff field, by moving water from dry upgradient (hilltop) to wetter downgradient (valley) locations. The end result of this landscape variability is to add smaller-scale spatial variability to the large-scale spatial forcing, thus resulting in a mixing of the spatial scales of the landscape and of the forcing and a reduction in the characteristic spatial scales from about $50 \mathrm{~km}$ for precipitation to about $10 \mathrm{~km}$ for groundwater. There is little reduction in the spatial scale of runoff compared with rainfall because the data used here is an integral over the catchment area. Back calculated characteristic space scales of hypothetical point runoff are indeed much smaller (about $1 \mathrm{~km}$ ). Note that the characteristic spatial scale of soil moisture is difficult to compare to the other fields due to the different spatial extent of the data sets. These decreases in spatial scale are more than an order of magnitude less than the increases in temporal scale, thus the changes in process velocity that are discussed below 
result more from temporal effects that are related to residence times and flow velocities through the various stores than from spatial effects.

[62] Most of the space-time traces of characteristic scales plot as straight lines or approximately straight lines in the double logarithmic diagram indicating that space-time scales are related by a power law of the form of equation (3). The scaling exponents $z$ found here are somewhat flatter than those shown in the schematic of Blöschl and Sivapalan [1995] and there are significant differences between the processes examined here. We will interpret the scaling exponents found here in the context of environmental transport processes. Taylor's [1938] hypothesis states that instead of measuring spatial variability one can measure temporal variability, and then calculate spatial variability by a simple conversion factor, the characteristic velocity. Taylor's hypothesis holds if the diffusion of the process is significantly smaller than advection, i.e., if a perturbation moves sufficiently fast through the sensor that it does not change too much during the time it passes the sensor. One way to express this relationship is the Peclet number which is the ratio of the advective term and the diffusive term in, say the groundwater advection dispersion equation. If the Peclet number is significantly larger than unity, then advection dominates and one would expect Taylor's hypothesis to hold. In the context of the present analysis one can replace spatial variability by temporal variability if and only if the spatial and temporal variograms of a particular process have the same shape. If they do have the same shape then $z$ in equation (3) must be $z=1$ as timescale and space scale must be proportional with a simple conversion factor, the characteristic velocity. We argue here that, conversely, if $z=1$ we can expect that advection dominates over diffusion. On the other hand if $z<1$ then one would assume that diffusion plays a significant role. Because of $z<1$ the characteristic velocity will increase with both space scale and timescale. This may be due to diffusive processes that come into play and contribute to changing the shape of a perturbation in addition to advection. The combined effect of advection and diffusion will give rise to larger characteristic velocities than advection alone.

[63] In the light of this reasoning we will interpret the $z$ values found here. Precipitation gives a relatively small value of $z=0.5$ implying, if one accepts the interpretation suggested above, that diffusion is quite important. For the space-time scales examined here this is likely, as the weather systems do change significantly within a day as they move over Austria, as evidenced by weather radar images. The value of $z$ is also consistent with the range of $z=0.5-0.6$, found from radar data analysis by FoufoulaGeorgiou and Vuruputur [2000] even though they worked at a timescale of minutes rather than days as is the case here. For groundwater, $z$ is significantly larger than the value for precipitation $(z=0.8)$, which may suggest that advection becomes relatively more important. All the groundwater level data used here stem from porous aquifers with significant lateral movement. Some of the aquifers are in mountain valleys and the water flow follows the topographic gradient. Other aquifers such as the Südliches Wiener Becken aquifer south of Vienna, again, has relatively high flow velocities which result from significant recharge in the upstream part adjacent to the mountains but very little recharge in the lower flatter part of it. Soil moisture on the other hand has a much smaller value of $z=0.4-0.5$, suggesting that advection is relatively unimportant as compared to diffusion. This is consistent with field observations in some of these catchments, which indeed suggest that the lateral redistribution of soil moisture is limited to a relatively short time of the year [Grayson et al., 1997]. For the case of catchment runoff, $z$ differs with catchment size class. For the smallest size class $z=0.8$, implying that advection is relatively more important than diffusion as compared to precipitation. As catchment size increases, so does $z$, implying that the importance of advection over that of diffusion increases even more. This can be related to the longer stream distances and stream routing times in larger catchments, which would suggest that they more strongly impose the characteristics of advection on the space-time variability of runoff than is the case in smaller catchments. One can also speculate that if one extrapolates the runoff traces in Figure 7 from large catchments beyond small catchments to give a trace for hypothetical point runoff, this trace would be flatter, perhaps with a slope of $z=0.5-0.6$. This slope is consistent with diffusion being quite important relative to advection, which is what one would expect for hypothetical point runoff. However, this reasoning is somewhat speculative as it does not explain the $z$ values larger than unity for large catchment runoff. Although the interpretations offered above are of a general nature, it is believed that they may contribute to a better understanding of macroscale spacetime hydrologic variability in the spirit of Dooge [1986].

\subsection{Outlook}

[64] The main strength of this paper, we believe, is that space and time variograms have been examined over many orders of magnitude, which is needed for a robust analysis of the presence of characteristic scales. This has been made possible by using a relatively comprehensive data set for each variogram. However, the robustness has been at the cost of lumping together a large number of stations for the temporal variograms, and a large number of time steps for the spatial variograms. This implies that many different subprocesses have been lumped together. Ideally one would like to derive characteristic scales for individual processes separately, in order to be able to link process and scale more closely. One example of this classification in the context of scale is given by Skaugen [1997] who classified precipitation events into two groups according to their spatial characteristics. He then calculated variograms for each of these groups separately. The groups had quite contrasting properties. One of the groups exhibited stationary variograms with characteristic scales on the order of $10 \mathrm{~km}$ while the other group exhibited nonstationary variograms with characteristic scales of $100 \mathrm{~km}$ or more. A process classification for runoff and catchment state has been suggested by Blöschl et al. [2000] which should be applicable to the problem studied here and will be pursued in future work.

[65] Another obvious extension of the work in this paper is to examine space-time aggregation effects. Although we were able to fit spatially aggregated runoff variograms to catchments of different size classes in a consistent way, a more complete approach would involve both spatial and 
temporal aggregation effects by making use of catchment size and catchment response times, respectively. For spacetime regularization more elaborate space-time variogram models are needed such as those suggested by De Cesare et al. [2001] and Christakos [2000].

[66] Some initial attempts at making use of characteristic scales of hydrologic processes as those derived in this paper do already exist. One example is network planning and the examination of scale effects in the context of soil moisture measurements [Western and Blöschl, 1999]. However, overall, we are still at a very early stage as compared to other disciplines. It is hoped that the characteristic scales of hydrologic processes as derived in this paper will contribute to fostering a more wide spread use of characteristic scales in hydrology.

[67] Acknowledgments. Our research work has been supported financially by the Austrian Academy of Sciences project HOE18, and the Cooperative Research Centre for Catchment Hydrology, Australian Research Council (projects C39804872, A39801842). We would like to thank the Hydrographic Office at the Federal Ministry of Agriculture, Forestry, Environment and Water Management in Austria for providing the Austrian data. We gratefully acknowledge the highly constructive comments of two anonymous reviewers.

\section{References}

Ambroise, B., La Dynamique du Cycle de l'Eau Dans un Bassin Versant, 200 pp., Editions HGA, Bucharest, Romania, 1999.

Anderson, M. G., and T. P. Burt, Subsurface runoff, in Process Studies in Hillslope Hydrology, edited by M. G. Anderson and T. P. Burt, pp. $365-$ 400, John Wiley, Hoboken, N. J., 1990.

Austin, P. M., and R. A. Houze, Analysis of the structure of precipitation patterns in New England, J. Appl. Meteor., 11, 926-935, 1972.

Blöschl, G., Scale and scaling in hydrology, Wien. Mitt. Wasser Abwasser Gewaesser, 132, 346 pp., 1996.

Blöschl, G., Scaling issues in snow hydrology, Hydrol. Processes, 13, 2149-2175, 1999

Blöschl, G., Scaling in hydrology, Hydrol. Processes, 15, 709-711, 2001.

Blöschl, G., and M. Sivapalan, Scale issues in hydrological modelling-A review, Hydrol. Processes, 9, 251-290, 1995.

Blöschl, G., and M. Sivapalan, Process controls on regional flood frequency: Coefficient of variation and basin scale, Water Resour. Res. 33(12), 2967-2980, 1997

Blöschl, G., R. B. Grayson, and M. Sivapalan, On the representative elementary area (REA) concept and its utility for distributed rainfall-runoff modelling, Hydrol. Processes, 9, 313-330, 1995.

Blöschl, G., R. Merz, D. Gutknecht, and M. Bachhiesl, Regional hydrological types-A tool for improved runoff forecasts, paper presented at XXth Conference of the Danubian Countries on Hydrological Forecasting and the Hydrological Basis of Water Management, SHMÚ Bratislava, Bratislava, Slovakia, 4-8 Sept. 2000

Buchholz, O., Hydrologische Modelle-Theorie der Modellbildung und Beschreibungssystematik, Mitteilungen des Lehrstuhls fü r Wasserbau und Wasserwirtschaft, 300 pp., RWTH Aachen, Verlag Mainz, Wissenschaftsverlag Aachen, Aachen, Germany, 2000.

Charney, J. G., On the scale of atmospheric motions, Geophys. Norv., 17(2), 4-17, 1948 .

Christakos, G., Modern Spatiotemporal Geostatistics, 288 pp., Oxford Univ. Press, New York, 2000.

De Cesare, L., D. E. Myers, and D. Posa, Estimating and modeling spacetime correlation structures, Stat. Prob. Lett., 51, 9-14, 2001.

De Cesare, L., D. E. Myers, and D. Posa, FORTRAN programs for spacetime modeling, Comput. Geosci., 28, 205-212, 2002.

Dooge, J. C. I., Linear theory of hydrologic systems, U.S. Dep. Agric. Tech. Bull., 1468,315 pp., 1973.

Dooge, J. C. I., Looking for hydrologic laws, Water Resour. Res., 22, 46S$58 \mathrm{~S}, 1986$

Dunne, T., Field studies of hillslope flow processes, in Hillslope Hydrology, edited by M. J. Kirkby, pp. 227-293, John Wiley, Hoboken, N. J., 1978.

Entin, J. K., A. Robock, K. Y. Vinnikov, S. E. Hollinger, S. X. Liu, and A. Namkhai, Temporal and spatial scales of observed soil moisture variations in the extratropics, J. Geophys. Res., 105, 11,865-11,877, 2000.
Fischer, H. B., E. J. List, R. C. Y. Koh, J. Imberger, and N. H. Brooks, Mixing in Inland and Coastal Waters, 483 pp., Academic, San Diego, Calif., 1979.

Fleming, S. W., A. M. Lavenue, A. H. Aly, and A. Adams, Practical applications of spectral analysis to hydrologic time series, Hydrol. Processes, 16, 565-574, 2002.

Fortak, H., Meterologie, 293 pp., Dietrich Reimer, Berlin, 1982.

Foufoula-Georgiou, E., and V. Vuruputur, Patterns and organisation in precipitation, in Spatial Patterns in Catchment Hydrology: Observations and Modelling, edited by R. Grayson and G. Blöschl, pp. 82104, Cambridge Univ. Press, New York, 2000.

Gallant, J. C., I. D. Moore, M. F. Hutchinson, and P. E. Gessler, Estimating fractal dimension of profiles: A comparison of methods, Math. Geol., 26, 455-481, 1994.

Gelhar, L. W., Stochastic Subsurface Hydrology, 390 pp., Prentice-Hall, Old Tappan, N. J., 1993.

Ghosh, B., Random distances within a rectangle and between two rectangles, Bull. Calcutta Math. Soc., 43, 17-24, 1951

Gottschalk, L., Correlation and covariance of runoff, Stochastic Hydrol. Hydraul., 7, 85-101, 1993.

Grayson, R. B., A. W. Western, F. H. S. Chiew, and G. Blöschl, Preferred states in spatial soil moisture patterns: Local and non-local controls, Water Resour. Res., 33, 2897-2908, 1997.

Gupta, V. K., and E. C. Waymire, Multiscaling properties of spatial rainfall and river flow distributions, J. Geophys. Res., 95, 1999-2009, 1990

Haltiner, G. J., and R. T. Williams, Numerical Prediction and Dynamic Meteorology, 477 pp., John Wiley, Hoboken, N. J., 1980.

Harte, J., Consider a Spherical Cow: A Course in Environmental Problem Solving, 283 pp., Univ. Sci. Books, Sausalito, Calif., 1988.

Hatcher, B. G., J. Imberger, and S. V. Smith, Scaling analysis of coral reef systems: An approach to problems of scale, Coral Reefs, 5, 171-181, 1987

Hurst, H. E., Long-term storage capacity of reservoirs, Trans. Am. Soc. Civ. Eng., 116, 770-808, 1951.

Isaaks, E. H., and R. M. Srivastava, An Introduction to Applied Geostatistics, 561 pp., Oxford Univ. Press, New York, 1989.

Katsev, S., and I. L'Heureux, Are Hurst exponents estimated from short or irregular time series meaningful?, Comput. Geosci., in press, 2003

Kirchner, J. W., X. Feng, and C. Neal, Fractal stream chemistry and its implications for contaminant transport in catchments, Nature, 403, 524527, 2000.

Klemeš, V., The Hurst phenomenon: A puzzle?, Water Resour. Res., 10, $675-688,1974$.

Koltermann, C. E., and S. M. Gorelick, Heterogeneity in sedimentary deposits: A review of structure-imitating, process-imitating, and descriptive approaches, Water Resour. Res., 32, 2617-2658, 1996.

Leopold, L. B., and T. Maddock, The hydraulic geometry of stream channels and some physiographic implications, U.S. Geol. Surv. Prof. Pap., 252, 57 pp., 1953.

Lovejoy, S., and D. Schertzer, Generalized scale invariance and fractal models of rain, Water Resour. Res., 21, 1233-1250, 1985.

Melone, F., C. Corradini, and V. P. Singh, Lag prediction in ungauged basins: An investigation through actual data of the upper Tiber River valley, Hydrol. Processes, 16, 1085-1094, 2002.

Miller, E. E., and R. D. Miller, Physical theory of capillary flow phenomena, J. Appl. Phys., 27, 324-332, 1956.

Milly, P. C. D., and R. T. Wetherald, Macroscale water fluxes: 3. Effects of land processes on variability of monthly river discharge, Water Resour. Res., 38(11), 1235, doi:10.1029/2001WR000761, 2002.

Niehoff, D., Modellierung des Einflusses der Landnutzung auf die Hochwasserentstehung in der Mesoskala, doctoral dissertation, 144 pp., Univ. Potsdam, Potsdam, Germany, 2002.

Orlanski, I., A rational subdivision of scales for atmospheric processes, Bull. Am. Meteorol. Soc., 56, 527-530, 1975.

Prandtl, L., Bericht über Untersuchungen zur ausgebildeten Turbulenz, Z. Angew. Math. Mech., 5, 136-139, 1925.

Quattrochi, D. A., GCIP studies in the large scale area (1sa) east (19971998): A discussion paper, GEWEX Continental-scale International Project, Natl. Oceanic and Atmos. Admin, Silver Spring, Md., 1997. (Available at http://wwwghcc.msfc.nasa.gov/GCIP/)

Rodríguez-Iturbe, I., Scale of fluctuation of rainfall models, Water Resour. Res., 22, 15S-37S, 1986.

Rodríguez-Iturbe, I., G. K. Vogel, R. Rigon, D. Entekhabi, F. Castelli, and A. Rinaldo, On the spatial organization of soil moisture fields, Geophys. Res. Lett., 22, 2757-2760, 1995. 
Roth, K., H. Flühler, W. A. Jury, and J. C. Parker, (Eds.), Field-Scale Water and Solute Flux in Soils, 294 pp., Birkhäuser Boston, Cambridge, Mass., 1990.

Schulze, R., Transcending scales of space and time in impact studies of climate and climate change on agrohydrological responses, Agric. Ecosyst. Environ., 82, 185-212, 2000.

Seed, A. W., R. Srikanthan, and M. Menabde, A space and time model for design storm rainfall, J. Geophys. Res., 104, 31,623-31,630, 1999.

Seyfried, M. S., and B. P. Wilcox, Scale and the nature of spatial variability: Field examples having implications for hydrologic modeling, Water Resour. Res., 31, 173-184, 1995.

Sivapalan, M., Scale problems in rainfall, infiltration and runoff production, Ph.D. dissertation, 271 pp., Dep. of Civ. Eng., Princeton Univ., Princeton, N. J., 1986

Sivapalan, M., and G. Blöschl, Transformation of point rainfall to areal rainfall: Intensity-duration-frequency curves, J. Hydrol., 204, 150-167, 1998.

Skaugen, T., Classification of rainfall into small- and large-scale events by statistical pattern recognition, J. Hydrol., 200, 40-57, 1997.

Smagorinsky, J., Global atmospheric modelling and the numerical simulation of climate, in Weather and Climate Modification, edited by W. N. Hess, pp. 633-686, John Wiley, Hoboken, N. J., 1974.

Sokal, R. R., and F. J. Rohlf, Biometry, 887 pp., W. H. Freeman, New York, 1995.

Stommel, H., Varieties of oceanographic experience, Science, 139, $572-$ $576,1963$.

Stull, R. B., An Introduction to Boundary Layer Meteorology, 666 pp., Kluwer Acad., Norwell, Mass., 1988.

Taylor, G. I., Diffusion by continuous movements, Proc. London Math. Soc., 20, 196-211, 1921

Taylor, G. I., The spectrum of turbulence, Proc. R. Soc. London, Ser. A, $164,476-490,1938$

Uchida, T., K. Kosugi, and T. Mizuyama, Effects of pipeflow on hydrological process and its relation to landslide: A review of pipeflow studies in forested headwater catchments, Hydrol. Processes, 15, 2151-2174, 2001.

Vanmarcke, E., Random Fields: Analysis and Synthesis, 382 pp., MIT Press, Cambridge, Mass., 1983.

Webster, R., and M. Oliver, Geostatistics for Environmental Scientists, 271 pp., John Wiley, Hoboken, N. J., 2002.
Western, A. W., and G. Blöschl, On the spatial scaling of soil moisture, J. Hydrol., 217, 203-224, 1999

Western, A. W., and R. B. Grayson, The Tarrawarra data set: Soil moisture patterns, soil characteristics and hydrological flux measurements, Water Resour. Res., 34(10), 2765-2768, 1998.

Western, A. W., G. Blöschl, and R. B. Grayson, Geostatistical characterisation of soil moisture patterns in the Tarrawarra catchment, J. Hydrol. $205,20-37,1998$

Western, A. W., R. B. Grayson, G. Blöschl, G. R. Willgoose, and T. A McMahon, Observed spatial organization of soil moisture and its relation to terrain indices, Water Resour. Res., 35, 797-810, 1999.

Western, A., R. Grayson, and G. Blöschl, Scaling of soil moisture: A hydrologic perspective, Annu. Rev. Earth Planet. Sci., 30, 149-180, 2002

Wilby, R. L., Contemporary Hydrology, 354 pp., John Wiley, Hoboken, N. J., 1997.

Woods, R., and M. Sivapalan, A synthesis of space-time variability in storm response: Rainfall, runoff generation, and routing, Water Resour. Res., $35,2469-2485,1999$

Woods, R., M. Sivapalan, and M. Duncan, Investigating the representative elementary area concept: An approach based on field data, Hydrol. Processes, 9, 291-312, 1995

Woods, R. A., R. B. Grayson, A. W. Western, M. J. Duncan, D. J. Wilson, R. I. Young, R. P. Ibbitt, R. D. Henderson, and T. A. McMahon, Experimental design and initial results from the Mahurangi River Variability Experiment: MARVEX, in Land Surface Hydrology, Meteorology and Climate, Water Sci. Appl. Ser., vol. 3, edited by V. Lakshmi, J. D. Albertson, and J. Schaake, pp. 201-213, AGU, Washington, D. C. 2001.

G. Blöschl and J. O. Skøien, Institut für Hydraulik, Gewässerkunde und Wasserwirtschaft, Technische Universität Wien, A-1040Wien, Austria. (bloeschl@hydro.tuwien.ac.at)

A. W. Western, Centre for Environmental Applied Hydrology and Cooperative Research Centre for Catchment Hydrology, Department of Civil and Environmental Engineering, University of Melbourne, Parkville, VIC 3010, Australia. 


\section{University Library}

\section{- M M I E E R VA A gateway to Melbourne's research publications}

Minerva Access is the Institutional Repository of The University of Melbourne

Author/s:

Skoien, JO;Bloschl, G;Western, AW

Title:

Characteristic space scales and timescales in hydrology

Date:

2003-10-30

Citation:

Skoien, J. O., Bloschl, G. \& Western, A. W. (2003). Characteristic space scales and timescales in hydrology. WATER RESOURCES RESEARCH, 39 (10), https:// doi.org/10.1029/2002WR001736.

Persistent Link:

http://hdl.handle.net/11343/297417 